DOE/ET-0074

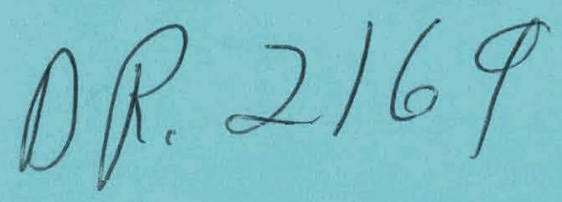

\title{
Lie Transforms \\ and Their Use in \\ Hamiltonian Perturbation Theory
}

June 1978

U.S. Department of Energy

Assistant Secretary for Energy Technology

Division of Applied Plasma Physics

Published January 1979

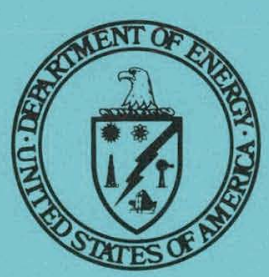




\section{DISCLAIMER}

This report was prepared as an account of work sponsored by an agency of the United States Government. Neither the United States Government nor any agency Thereof, nor any of their employees, makes any warranty, express or implied, or assumes any legal liability or responsibility for the accuracy, completeness, or usefulness of any information, apparatus, product, or process disclosed, or represents that its use would not infringe privately owned rights. Reference herein to any specific commercial product, process, or service by trade name, trademark, manufacturer, or otherwise does not necessarily constitute or imply its endorsement, recommendation, or favoring by the United States Government or any agency thereof. The views and opinions of authors expressed herein do not necessarily state or reflect those of the United States Government or any agency thereof. 


\section{DISCLAIMER}

Portions of this document may be illegible in electronic image products. Images are produced from the best available original document. 


\section{Lie Transforms and Their Use in \\ Hamiltonian Perturbation Theory}

June 1978

Prepared by:

John R. Cary

Lawrence Berkeley Laboratory ${ }^{\prime \prime \prime}$

For

U.S. Department of Energy

Assistant Secretary for Energy Technology

Division of Applied Plasma Physics

Washington, D.C. 20545

This report was prepared as an account of work Sponsored by the United States Govermment. Neither the Energy, nor any of their employees, nor any of their contractors, subcontractors, or their employees, makes any warranty, express or implied, or assumes any legal liability or responsibility for the accuracy, completeness of use fulness of any information, apparatus, product or process disclosed, of represents that its use would not infringe privately owned rights.

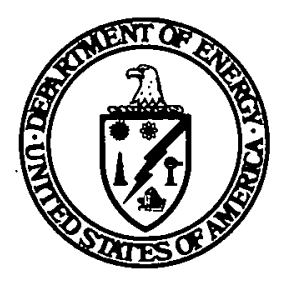


Available from:

National Technical Information Service (NTIS)

U.S. Department of Commerce 5285 Port Royal Road

Springfield, Virginia 22161

Price: Printed Copy: $\quad \$ 5.25$

Microfiche: $\$ 3.00$ 
LIE TRANSFORMS AND THEIR USE IN HAMILTONIAN PERTURBATION THEORY*

\author{
John R. Cary ${ }^{a)}$ \\ Lawrence Berkeley Laboratory, University of California \\ Berkeley, California 94720.
}

\begin{abstract}
We present a review of the theory of Lie transforms as applied to Hamiltonian systems. We begin by presenting some general background on the Hamiltonian formalism and by. Introducing the operator notation for canonical transformations. We then derive the general theory of Lle transforms. We derive the formula for the new Hamiltonian when one uses a Lie transform to effect a canonical transformation, and we use Lie transforms to prove a very general version of Noether's theorem, or the symmetry-equals-invariant theorem. Next we use the general Lie transform theory to derive Deprit's perturbation theory. We illustrate this perturbation theory by application to two well known problems in classical mechanics. Finally we present a chapter on conventions. There are many ways to develop lie transforms. The last chapter explains the reasons for the choices made here.

a) Present address: Los Alamos Scientific Laboratory, Theoretical Division, Los Alamos, New Mexico 87545.

*Work performed under the auspices of the U.S. Department of Energy.
\end{abstract}


TABLE OF CONTENTS

1. Introduction

2. Notation and Background

2.1 Canonical Transformations

2.2 The Time Development Operator

2.3 Short Time Solution for the Time Development Operator; Secular Perturbation Theory

3. Continuous Families of Canonical Transformations

3.1 The Generating Function.

3.2 The New Hamiltonian

3.3 An Illustration

3.4 The Symmetry-Equals-Invariant Theorem (Noether's Theorem)

4. Deprit Perturbation Theory

4.1 Deprit Perturbation Series Relations

4.2 The Anharmonic Oscillator

4.3 The Parametrically Driven Anharmonic Oscillator

4.4 Superconvergence; Kolmogorov's Technique

5. Discussion of the Choice of Conventions

6. Acknowledgements

Appendix A. Notations for Derivatives 


\section{Introduction}

Canonical transformations have long been used for finding the solutions (or trajectories) of a given Hamiltonian. In Hamilton-Jacobi theory [1-3] one is searching for the generating function of mixed variables $F(q, P, t)$ which effects a transformation to a system where the Hamiltonian vanishes, and is therefore trivially solved. However, solving the Hamilton Jacobi equation is too difficult except in the simplest of systems, and so we must turn to perturbation theory.

In perturbation theory, one considers a Hamiltonian which consists of a solvable term and an small additional term. By small we mean that the effects of the term are small, so that the motion with the perturbation does not differ greatly from the motion without the perturbation. If this is the case, one can hope to find a small canonical transformation which transforms away the perturbation. In practice one only finds the transformation to some order in a small parameter. If a transformation is canonical only to some order in a small parameter, we call it an infinitesimal canonical transformation.

In general, all Hamiltonian perturbation theories rely on some method for performing infinitesimal canonical transformations. The oldest is the Poincare-Von Zelpel method $[4,5]$. This method consists of a "brute force" ordering of the function $F(q, P, t)$, the varlable transformation, and the Hamilton-Jacobi equation. A variant of this method was found by Kolmogorov [6]. By performing successive small transformations rather than a single transformation; Kolmogorov found quadratic convergence. This means that the Hamiltonian can be transformed away successively to orders $\varepsilon, \varepsilon^{2}, \varepsilon^{4}, \varepsilon^{8}, \ldots$, rather than the usual $\varepsilon, \varepsilon^{2}, \varepsilon^{3}, \varepsilon^{4} \ldots$. 
A great improvement in Hamiltonian perturbation theory was brought about by the introduction of Lie transforms by Hor1 [7]. The Lie transform technique is simpler since no functions of mlxed variables appear, and all of the terms are repeated Poisson brackèts, making this theory canonically' invariant. Deprit [8] improved this scheme by defining the transformation in a different way, which allowed him to find recursion relations'for terms in the expansion of the transformation: The Lie transform technique can also be combined with Kolmogorov's method of successive transformations to obtain quadratic convergence [9].

One drawback of Deprit's work is that the transformation theory is entirely expressed in terms of power series. Dewar [10] eliminated this: drawback by developing a transformation theory in which the transformations can be written in closed form. As a result of Dewar's work, the overall structure of the theory is seen, and the theory is more easily understood. Dewar [11] has also used this theory to study a case where the transformation is not expandable in a power series.

The main purpose of this paper is to explain the use of Lie transforms in Hamiltonian perturbation theory. We present the operator theory of Dewar, and from it we derive the Deprit perturbation series: We illustrate the various objects in the theory with examples. We present the perturbation equations to fourth order, and we do some explicit perturbation calculations.

We begin in chapter 2, by discussing general Hamiltonian theory. We give an operator notation for the time development of a canonical system. We relate the time development transformation to the Hamiltonian, and we give the formal expression for the time development transformation analogous to the formula for the time development transformation of quantum mechanics ' in terms of time ordered exponentials [12]. 
We next develop Dewar's transformation theory in analogy with the time development operator. Dewar introduces a transformation which depends on a parameter we call $\theta$. The time development transformation depends on the parameter $t$. (time).

In Chapter 4, we derive the Deprit perturbation theory from Dewar's equations, and we apply the Deprit perturbation theory to some problems to 1llustrate its use: We also show how superconvergence can arise in Deprit perturbation theory, and we discuss its relevance in practical calculations.

In writing this paper, we have chosen some conventions different from those of previous authors. In Chapter 5 we discuss the reason for these choices, and in the process we compare our choices to those of previous authors. 
2. Notation and Background

In this chapter we lay the foundation for later work. We briefly discuss: the elements of Hamiltonian mechanics: Polsson brackets and canonical transformations. This discussion is not intended as a substitute for those found in the textbooks on this subject $[1-3,13,14]^{\circ}$. It is mostiy a repetition of standard material, but rewritten using operator notation, which proves very convenient for discussing Lie transforms.

Using the operator notation we discuss the time development transformation generated by a Hamiltonian. We show how it can be used to solve Liouville's equation. Finally we discuss how it can be found using secular perturbation theory, In analogy with quantum mechanics. 


\subsection{Canonical Transformations}

We work in a $2 \mathrm{~N}$ dimensional space. The variables are collectlvely denoted by the vector $z_{.}$. The components of $z$ are $z_{1}, \ldots, z_{N}=q_{1}, \ldots, q_{N}$ and $z_{N+1}, \ldots, z_{2 N}=p_{1}, \ldots, p_{N}$. The Poisson bracket relations are contained in the matrix $\gamma$ defined by

$$
\gamma_{i j} \equiv\left\{z_{i}, z_{j}\right\}=\left\{\begin{array}{l}
1 \text { for } j=1+N \\
-1 \text { for } i=j+N \\
0 \text { otherwise } \\
\cdots
\end{array}\right.
$$

By inspection we see that the matrix $\gamma$ is antisymetric and invertible:

$$
\begin{aligned}
\gamma_{i j} & =-\gamma_{j i} \\
-\sum_{j} \gamma_{i j} \gamma_{j k} & =\delta_{i k}
\end{aligned}
$$

We now consider a time dependent mapping of the $2 \mathrm{~N}$ variables by the vector function $\underset{\sim}{Z}(\underset{\sim}{z}, t)$. We call this mapping canonical if the new variables have the same Poisson bracket relations as the old. The Poisson bracket of two functions $f$ and $g$ is generally given by

$$
\{f, g\}=\sum_{i=1}^{N} \frac{\partial f}{\partial q_{i}} \frac{\partial g}{\partial p_{i}}-\frac{\partial f}{\partial p_{i}} \frac{\partial g}{\partial q_{i}}=\sum_{\substack{i=1 \\ j=1}}^{2 N} \frac{\partial f}{\partial z_{i}} \gamma_{i j} \frac{\partial g}{\partial z_{j}} .
$$

Thus, the mapping $\underset{\sim}{Z}(\underset{\sim}{z}, t)$ is canonical if

$$
\left\{z_{i}, z_{j}\right\}=\left\{z_{i}, z_{j}\right\}
$$

or

$$
\sum_{k, l} \frac{\partial z_{i}}{\partial z_{k}} \gamma_{k \ell} \frac{\partial z_{j}}{\partial z_{\ell}}=\gamma_{i j}
$$

We take it to be well known that a canonical mapping preserves the Hamiltonian equations of motion. 
Given any canonical mapping $\underset{\sim}{z}(\underset{\sim}{z}, t)$ of the phase space variables, we define a corresponding canonical transformation operator $T(t)$ which operates on functions of phase space. The action of this operator is that it evaluates the function at the mapped point. If $\mathrm{f}=\mathrm{Tg}$, then

$$
f(z, t) \equiv g(\underset{\sim}{z}(\underset{\sim}{z}, t), t) \text {. }
$$

A particular example of this is when $g(z, t)=z_{1}$, one of the canonical variables. (Strictly speaking we mean the function $z_{i}$, not the variable; if there were only one variable $z$, this would be the identity function, $g(z)=z$.) By our rule for $\mathrm{Tg}$, we have

$$
T \underset{\sim}{z}=\underset{\sim}{Z}(z, t)
$$

We see that the mapping is obtained by applying the transformation to the variables (but thought of as functions).

We also introduce an operator to denote the Poisson bracket. To every phase space function $f(z, t)$ we assign an operator $L_{f}$ whose action on a function $g$ is given by

$$
\mathrm{L}_{\mathrm{f}} \mathrm{g}=\{\mathrm{f}, \mathrm{g}\}
$$

Some authors have defined $\mathrm{L}_{\mathrm{f}}$ with a minus sign relative to this choice. We have made this choice so that Jacobi's identity in operator form is easy to remember :

$$
\left[\mathrm{L}_{f}, \mathrm{~L}_{g}\right]=\mathrm{L}_{\{f, g\}}
$$

In the above equation the square brackets denote the commutator of the two operators.

We note that the mapping $\mathrm{f} \rightarrow \mathrm{L}_{\mathrm{f}}$ is linear and unique, but it is not invertible. If we are given two operators satisfying $\mathrm{L}_{f}=\mathrm{L}_{g}$, we cannot conclude that $\mathrm{f}=\mathrm{g}$. The statement $\mathrm{L}_{f}=\mathrm{L}_{g}$ is equivalent to saying that $\mathrm{L}_{f} z_{i}=\mathrm{L}_{\mathrm{g}} \mathrm{z}_{i}$ for all $i$. Hence, from the equality of $\mathrm{L}_{f}$ and $\mathrm{L}_{g}$ we can deduce only that

$$
\frac{\partial f}{\partial z_{j}}=\frac{\partial g}{\partial z_{j}} \text { for } a 1 i_{j} \text {. }
$$


Thus we conclude that the difference $(f-g)$ has no functional dependence on the canonical variables, but it may depend on time or other parameters.

To illustrate how the use of the operator notation simplifies equations, we consider the statement that Poisson bracket relations are preserved by canonical transformations. In the non operator notation the equation is written

$$
\{f[\underset{\sim}{Z}(\underset{z}{z}, t), t], g[\underset{\sim}{Z}(\underset{\sim}{z}, t), t]\}=\left.\{f, g\}\right|_{\underset{\sim}{Z}(\underset{\sim}{z}, t), t} .
$$

In words, transforming the functions and then forming the Poisson bracket gives the same result as first forming the Poisson bracket and then transforming. In operator notation this statement is written

$$
\mathrm{L}_{\mathrm{Tf}} \mathrm{T} \cdot \mathrm{g}=\mathrm{TL}_{\mathrm{f}} \mathrm{g}
$$

Since this is true for any $g$, we have the operator relation,

$$
\mathrm{TL}_{\mathrm{f}}=\mathrm{L}_{\mathrm{Tf}} \mathrm{T} \text { or } \mathrm{TL}_{\mathrm{f}} \mathrm{T}^{-1}=\mathrm{L}_{\mathrm{Tf}} \text {, }
$$

for any canonical transformation $T$, and any phase space function $f$.

When evaluating a Poisson bracket, such as the one on the left of (2.1.11), we will need to use the chain rule to calculate derivatives. To avoid confusion, we introduce a very explicit notation for derivatives. By the symbol

$$
\left.\frac{\partial g}{\partial z}\right|_{z(z, t), t,}
$$

we mean: take the derivative of the function $g(z, t)$ with respect to the variable $z_{m}$, then substitute $\left.\underset{\sim}{Z} \underset{\sim}{z}, t\right)$ and $t$ into the argument slots of this new function. We use this cumbersome notation since it is unambiguous as explained in Appendix A. To illustrate the use of this notation we apply the chain rule to Eq. $(2.1 .6)$ : 


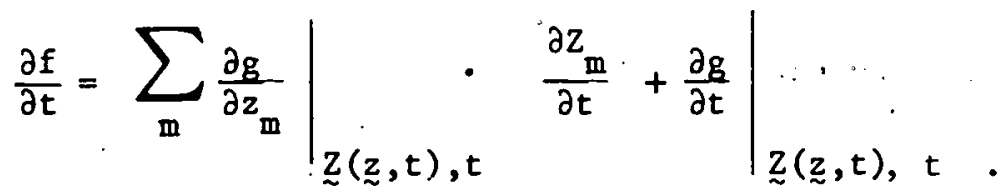

Whenever we do not explicitly show the arguments of a function, they are assumed to be $(z, t)$.

Finally, we want to introduce the concept of an infinitesimal canonical transformation. Such a transformation is of the form

$$
T=I+\varepsilon L_{g}
$$

where $g$ is any phase function, and $\varepsilon$ is near zero. This transformation is called an infinitesimal canonical transformation of order $\varepsilon$, since it is canonical to order $\varepsilon^{2}$. To see this, consider the Poisson bracket of $\left\{z_{i}(z, t), z_{j}(z, t)\right\}$. It is given by

$$
\begin{aligned}
\left\{z_{i}(\underset{z}{z}, t), z_{j}(\underset{z}{z}, t)\right\}= & \left\{z_{i}, z_{j}\right\}+\varepsilon\left\{z_{i},\left\{g, z_{j}\right\}\right\} \\
& +\varepsilon\left\{\left\{g, z_{i}\right\}, z_{j}\right\}+\theta\left(\varepsilon^{2}\right) .
\end{aligned}
$$

Using (2.1.1), and Jacobi's identity, this can be put in the form

$$
\left\{z_{i}, z_{j}\right\}=\gamma_{i j}+\theta\left(\varepsilon^{2}\right) \text {, }
$$

and is therefore canonical to order $\varepsilon^{2}$. 


\subsection{The Time Development Operator}

In Hamiltonian mechanics we solve for the trajectories of a given Hamiltonian. A trajectory is found by starting at some position $z_{0}$, and integrating forward in time ușing Hamilton's equations, $\underset{\sim}{\dot{z}}=\{\underset{\sim}{z}, \mathrm{~h}\}$. An alternative point of view is that Hamilton's equations specify a mapping $z(z, t)$, which gives the position of a particle at time $t$ for a particle which was at $\underset{\sim}{z}$ at $t=0$. The equations which define the mapping are

and

$$
\frac{\partial \eta_{i}}{\partial t}=\left.\sum_{j} \gamma_{i j} \frac{\partial h}{\partial z_{j}}\right|_{\underset{z}{z}(z, t), t}
$$

$$
y_{i}(z, 0)=z_{i}
$$

If one knows the mapping, one knows all of the trajectories. Hamiltonian mechanics therefore consists of finding the mappings associated with given Hamiltonians .

In this section we develop this point of view. We start with this mapping and construct the corresponding transformation. We show how the time derivative of the transformation is related to the Hamiltonian. We show how this transformation can be used to solve the Inhomogeneous Liouville equation $(2,2.12)$. Finally we give an example of a time development transformation.

The importance of this section is that it formalizes the method of solving the inhomogeneous Liouville equation by integrating along particle orbits. We give the explicit solution in terms of the time development operator. Of course, our expression is useful only when the time development operator is explicitly known, i.e. for solvable Hamiltonians. However, this is a situation which occurs repeatedly in perturbation theory. In perturbation 
theory we have a solvable Hamiltonian $h_{0^{*}}$. The effects of the perturbation $\Delta h$ are found by integrations along the particle orbits of $h_{0}$.

One can show that the mapping $f_{f}(z, t)$ is canonical by showing that the time derivative of the Poisson bracket $\left\{y_{i}, y_{j}\right\}$ vanishes. We do not show this here since it is a standard topic in most texts (e.g. ref. [1] ch. 7). We call this mapping the time development mapping. The corresponding transformation $M(t)$ is the time development transformation. $M(t)$ is defined as follows. The relation $\mathrm{f}=\mathrm{Mg}$ means

$$
f(\underset{\sim}{z}, t)=g(\underset{z}{z} \underset{\sim}{z}, t), t) .
$$

The effect of the mapping $M$ is to develop the observables in time. As an example, suppose we are given the potential energy $V(z, t)$ at time $t$ as a function of the particle's position at time $t$. By applying $M$, we get a new function $?$ ? defined by

$$
\mathcal{Q}(\underset{\sim}{z}, t) \equiv(M V)(\underset{\sim}{z}, t)=v(\underset{z}{z}(\underset{\sim}{z}, t), t) .
$$

The new function 9 is the potential energy at time $t$ of a particle which was at phase point $z$ initially (i.e. at $t=0$ ). Another example is where $g=z_{i}$, one of the variables, but thought of as a function, as in Eq. (2.1.7). In this case $\mathrm{Mz}_{i}=Z_{i}(\underset{\sim}{z}, t)$.

Since the mapping $z(\underset{z}{z}, t)$ is related to $h$ by $(2.2 .1)$, we expect the time derivative of $M$ to be related to $h$. We define the time derivative of the operator $M$ by the usual procedure:

$$
\frac{\partial M}{\partial t} g \equiv \operatorname{Lim}_{\tau \rightarrow 0} \frac{M(t+\tau) g-M(t) g}{\tau} .
$$

This definition insures that the product rule for differentiation will hold, $\frac{\partial}{\partial t}(M g)=\frac{\partial M}{\partial t} g+M \frac{\partial g}{\partial t}$. In our case, where the action of $M$ is given by $(2.2 .4)$, we can use the chain rule to show that the time derivative of $M$ is given by 


$$
\left(\frac{\partial M}{\partial t} g\right) \underset{\sim}{(z, t)}=\left.\left.\sum_{i} \frac{\partial}{\partial z}\right|_{i}\right|_{j(z, t), t} \frac{\partial \gamma_{i}}{\partial t}
$$

Inserting (2.2.1) Into this expression, we get

$$
\left(\frac{\partial M}{\partial t} g\right)(z, t)=\left.\left.\sum_{i j} \frac{\partial g}{\partial \dot{z}_{i}}\right|_{\xi(z, t), t} \gamma_{i j} \frac{\partial h}{\partial \dot{z}_{j}}\right|_{\frac{3}{\gamma}(z, t), t} .
$$

Since $g$ is arbitrary, $(2.2 .7)$ is the same as the operator equality

$$
\frac{\partial M}{\partial t}=-M_{h} \cdot
$$

Whenever we are given a relation such as $(2.2 .8)$, we say that the transformation $M$ is generated by the function $h$. To see why, we use the expression $(M(t+\tau)-M(t)) / \tau+\sigma(\tau)$ for the time derivative of $M$ in equation (2.2.9).

The result,

$$
M(t+\tau)=M(t)\left(I-\tau L_{h}+\sigma\left(\tau^{2}\right)\right)
$$

Shows that we generate the operator $M(t+\tau)$ from the operator $M(t)$ by multiplication of $M(t)$ by the infinitesimal canonical transformation $I-\tau_{h}$

The inverse operator $\mathrm{M}^{-1}(t)$ has properties similar to those of $M$. We define $M^{-1}$ by the same route. We first consider the inverse mapping $z^{-1}(z, t)$, which gives the initial position of a particle which is at $z$ at time $t$. Then we define $M^{-1}$ by

$$
\left(M^{-1} g\right)(z, t) \equiv g\left(Z^{-1}(z, t), t\right) \text {. }
$$

By definition we have $M^{-1} M=M^{-1}=I$. By differentiating this expression we get

$$
\frac{\partial M^{-1}}{\partial t}=L_{h} M^{-1}
$$

In our discussion, the time $t=0$ has been a special time. However, we could have instead used time $t_{0}$ as the inftial time. The time development transformation from $t_{0}$ to $t$ is given by $M(t) M^{-1}\left(t_{0}\right)$, 1.e. we transform back 
from $t_{0}$ and then forward to $t$.

We can now show how to solve the inhomogeneous Liouville equation;

$$
\frac{\partial f}{\partial t}+\{f, h\}=g,
$$

with the operator $M$. The equation occurs over and over in perturbation theory. There the function $h$ is the unperturbed Hamiltonian, the function $g$ is the perturbation, and $f$, which we are solving for, defines the canonical transformation used in perturbation theory. To solve (2.2.12) we introduce the function $f^{\prime}=$ Mf. Using (2.2.8) we see that $f^{\prime}$ satisfies

$$
\frac{\partial f^{\prime}}{\partial t}=M g \text {. }
$$

This equation is now trivially integrated giving

$$
f^{\prime}(t)=\int_{t_{0}}^{t} d \tau M(\tau) g(\tau)+f^{\prime}\left(t_{0}\right) .
$$

Inserting $f^{\prime}=$ Mf into $(2.2 .14)$ gives

$$
f(t)=M^{-1}(t) \int_{t_{0}}^{t} \operatorname{d\tau M}(\tau) g(\tau)+M^{-1}(t) M\left(t_{0}\right) f\left(t_{0}\right)
$$

as the general solution of $(2.2 .12)$. This method of solution is commonly known as finding $f$ by integrating $g$ along the particle orbits of $h$.

A spectal case of particular importance is when $g=0$. In this case (2.2.12) reduces to the Llouville equation, and Its solution (from 2.2.15) is $\left(\right.$ for $\left.t_{0}=0\right)$ :

$$
f(t)=M^{-1}(t) f(0)
$$

or

$$
f(z, t)=f\left(z^{-1}(z, t), 0\right) .
$$

We see in (2.2.16) that to find a distribution at a later time, we apply. $M^{-1}$. That is, $M(t)$ is the time development operator for observables, but $M^{-1}(t)$ is the time development operator for distributions. 
When the Hamiltonian $h$ is time independent, many of the results simplify. In particular, $L_{h}$ and $M$ comute in this case.

$$
L_{h} M(t)=M(t) L_{h}
$$

To see this, we use the fact that the time development transformation preserves the Hamiltonian, $M(t) h:=h$, if $h$ is time independent. From this we have

$$
\mathrm{L}_{\mathrm{M}(\mathrm{t}) \mathrm{h}}=\mathrm{L}_{\mathrm{h}} \text { ? }
$$

which yields $(2.2 .17)$ upon insertion of $(2.1 .13)$.

Using $(2.2 .17)$ we can prove the relation

$$
M\left(t-t^{\prime}\right)=M(t) M^{-1}\left(t^{\prime}\right) \text {. }
$$

on physical grounds, this looks true. If $h$ is time independent, the transformation which develops observables from time 0 to time $t-t$ ', on the left side of Eq. (2.2.19), must be the same as the transformation which develops observables In time $t^{\prime}$ to $t^{\prime}+\left(t-t^{\prime}\right)$, the right side of Eq. (2.2.19). If $h$ is $t$ ime independent, $M$ can depend only on the time difference. Using (2.2.19), we can simplify $(2.2 .15)$. Inserting gives

$$
\begin{aligned}
f(t) & =\int_{t_{0}}^{t} d \tau M(\tau-t) g(\tau)+M^{-1}\left(t-t_{0}\right) f\left(t_{0}\right) \\
& =\int_{t_{0}-t}^{0} \operatorname{d\tau M}(\tau) g(t+\tau)+M^{-1}\left(t-t_{0}\right) f\left(t_{0}\right) .
\end{aligned}
$$

For an 1llustration of these ideas, we consider the free particle Hamiltonian $h=\frac{1}{2} p^{2}$ for one degree of freedom. Using (2.2.1), we see that the time development mapping is

$$
\begin{aligned}
& q(q, p, t)=q+p t \\
& f(q, p, t)=p .
\end{aligned}
$$

Now we consider the density function in $x$-space given a particle's phase space coordinate $(q, p): \rho(x, t ; q, p)=\delta(x-q)$. By applying the time development operator to $\rho$, we get a new function $\rho^{\prime}=M \rho$, 


$$
\rho^{\prime}(x, t ; q, p)=\delta(x-q-p t)
$$

Whereas $\rho$ gives the density at time $t$ as a function of the coordinate at time $t, \rho$ 'gives the density at time $t$ as a function of the initial $(t=0)$ phase space coordinates. One must always keep in mind that $z$ or $(q, p)$ are dummy variables.

Now suppose we have a particle distribution which is gaussian in $q$ and $p$ at $t=0$

$$
f(q, p, 0)=e^{-\frac{1}{2} p^{2}-\frac{1}{2} q^{2}}
$$

To find the distribution at time $t$, we develop $f$ in time using $M^{-1}$ as in (2.2.16). The inverse transformation is trivially solved for, $q^{-1}=q-p t$ and $p^{-1}=p$, so we get

$$
\begin{aligned}
f(q, p, t) & =M^{-1}(t) f(q, p, 0) \\
& =e^{-\frac{1}{2} p^{2}-\frac{1}{2}(q-p t)^{2}}
\end{aligned}
$$


2.3 Short Time Solution for the Time Development Operator; Secular Perturbation Theory.

The operator representation $M$ of the time development transformation of the previous section looks very simflar to the time development transformation $U$ in quantum mechanics [12]. In quantum mechanics the operator $U$ is unftary and its time derivative is found by multiplication by the antihermitian operator $-1 \mathrm{H}$,

$$
\frac{\partial U}{\partial t}=-\mathbf{i H U}
$$

In Hamiltonian mechanics the time derivative of the operator $M^{-1}$ is found by multiplication by the antihermitian operator $I_{h}$ as in (2.2.11). This analogy leads us to construct expressions for the time development operator $M$ in terms of time ordered products as in quantum mechanics.

We employ the method of successive approximations to find $M$. We assume $M$ is of the form

$$
M(t)=I+M_{1}(t)+M_{2}(t)+\ldots,
$$

where the terms are ordered in powers of the Hamiltonian. Inserting this into $(2.2 .8)$ gives

$$
\frac{\partial M_{n}}{\partial t}=-M_{n-1} L_{h}
$$

which is easily integrated, resulting in

$$
M_{n}(t)=-\int_{0}^{t} d \tau M_{n-1}(\tau) L_{h(\tau)}
$$

By repeating the process in (2.3.4) we arrive at the general formula for the $\mathrm{n}^{\text {th }}$ term:

$$
M_{n}=(-1)^{n} \int_{0}^{t} d t_{1} \int_{0}^{t_{1}} d t_{2} \cdots \int_{0}^{t_{n-1}} d t_{n} L_{h\left(t_{n}\right)} \cdots L_{h\left(t_{1}\right)} \cdots
$$


This expression is exactly analogous to the quantum mechanical expression. As in quantum mechanics, we can introduce the time ordering operator $\mathcal{J}^{\prime}$ which rearranges operators putting the one at earliest time first. Then the expression for $M_{\mathfrak{n}}$ becomes

$$
M_{n}=\frac{1}{n !} \mathcal{O}\left(-\int_{0}^{t} d \tau \tau_{h(\tau)}\right)^{n}
$$

allowing $M$ to be written

$$
M=\mathcal{J} \exp \left(-\int_{0}^{t} d \tau L_{h(\tau)}\right) \text {. }
$$

This form for $M$ is of course purely formal. In writing (2.3.7) we mean that one sums up the separate terms $M_{n}$ found by (2.3.5). Only in very special cases can this sum be done in closed form. An example is the time Independent Hamiltonian $\mathrm{H}=\frac{1}{2^{2}}$ for which we have

$$
M=\exp \left(t p \frac{\partial}{\partial q}\right)
$$

This is just the Taylor operator for a shift in $q$ by the amount tp.

$$
M f=g \Rightarrow g(q, p, t)=f(q+t p, p, t)
$$

In complicated cases, the sum is not known. Practicality requires that we evaluate only the first few terms of $M$, and hence we get a solution which is valid only for short times.

This same technique can be used to construct a perturbation theory. We consider the Hamiltonian $h=h_{0}+h_{1}$, where $h_{0}$ is the solved unperturbed Hamiltonian, and $h_{1}$ is a perturbation. If the effects of $h_{1}$ are small, then we expect the total time development transformation $M$ to differ little from the unperturbed time development transformation $M_{0}(t)$. Thus we write

$$
M(t)=m(t) M_{0}(t),
$$

and we expect $m(t)$ to be close to the identity. 
Differentiating $M$, and using the known time derivative of $M$, we find

$$
\frac{\partial M}{\partial t}=-M_{h}=\frac{\partial m}{\partial t} M_{0}+m \frac{\partial M_{0}}{\partial t}
$$

Now we use $I_{h}=L_{h}+L_{h}$ and the known time derivative of $M_{0}$ in $(2.3 .11)$. The result is

$$
\begin{aligned}
\frac{\partial \mathrm{m}}{\partial t} & =-m \mathrm{M}_{0} L_{h_{1}} M_{0}^{-1} \\
& =-m \mathrm{~L}_{\mathrm{M}_{0} h_{1}} .
\end{aligned}
$$

The last step follows from (2.1.12).

Noting the analogy between (2.3.12) and (2.2.8), we find $m$ just as we found $M$. The result is

$$
m=J^{\text {is }} \exp \left(-\int_{0}^{t} d \tau \mathrm{L}_{0}(\tau) \mathrm{h}_{1}(\tau)\right) \text {. }
$$

This form of perturbation theory is analogous to the Dyson Wick Feynman formalism in quantum mechanics. In quantum mechanics, the theory ultimately breaks down due to divergences. In classical mechanics the theory breaks down because of secular terms. In quantum mechanics, the divergences are regularized using a renormalization technique. In classical mechanics we treat the secular terms by isolating them. We use a transformation theory to get rid of rapidly oscillating terms, and we study the resonant terms Individually. The development of a convenient transformation theory is the subject of the remainder of this paper. 


\section{Continuous Families of Canonical Transformations}

We now consider canonical mappings which depend on an additional parameter $\theta$. That is, the mapping $\underset{\sim}{z}(\underset{\tau}{z}, t, \theta)$ is canonical for all values of $t$ and for $\theta$ in some domain. Further, we require that $\underset{\sim}{z}(\underset{\sim}{z}, t, \theta)$ be continuous, and twice differentiable in all variables simultaneously. We call this set of mappings (all $\theta$ values) a continuous family of canonical transformations.

In this chapter we will examine the consequences of the parametric dependence of $\underset{\sim}{Z}(\underset{\sim}{z} ; t, \theta)$. We first establish the fact that the mapping can be specified by a single function $w(z, t, \theta)$, in place of the $2 \mathrm{~N}$ functions $z(z, t, \theta)$. This fact is proven elsewhere $[1,16]$, but we include it here for completeness. In the second section, we derive the expression for the transformed system. For the case where $w$ is a power series in $\theta$, this was first done by Deprit [8]. Later, Dewar [10] found the general expression for the new Hamiltonian when $w$ is not a power series in $\theta$.

The last two sections are illustrations of the theory. Section 3 consists of a simple example where all of the operators can be found explicitly. In section 4 we use the theory to prove the symmetry-equals-invariant theorem, known as Noether's theorem in Lagrangian mechanics. 


\subsection{The Generating Function}

An important property of canonical transformations is that they can be specified by a single function. This property is helpful since it means that one can work with this single function rather than the $2 \mathrm{~N}$ functions $\underset{\sim}{Z}(\underset{\sim}{z}, t)$. In Hamilton-Jacobi theory this function is $F(q, P, t)[1,3]$, the generating function of mixed variables.

Given a family of canonical mappings $\underset{\sim}{Z}(\underset{\sim}{z}, t, \theta)$, we will show that there exists a function $w(\underset{\sim}{z}, t, \theta)$ satisfying

$$
\begin{aligned}
\frac{\partial z_{i}}{\partial \theta} & =\left\{z_{i}, w(\underset{\sim}{z}(\underset{\sim}{z}, t, \theta), t, \theta)\right\} \\
& =\left.\sum_{j} \gamma_{i j} \frac{\partial w}{\partial z_{j}}\right|_{z(\underset{\sim}{z}, t, \theta)}, t, \theta .
\end{aligned}
$$

This property is shown in ref. [1] sec. VII-2, but we include a proof for completeness. If $w$ has appropriate properties (say $w \in C_{\infty}$ ), we can specify $\underset{\sim}{Z}(z, t, \theta)$ as the unique solution of $(3.1 .1)$ for some given boundary conditions. In our work we will always require that the mapping reduce to the identity. when $\theta=0$ giving the boundary condition

$$
\underset{\sim}{Z}(\underset{\sim}{z}, t ; 0)=\underset{\sim}{z}
$$

We note that equation $(3.1 .1)$ is analogous to $(2.2 .1)$. Just as the Hamiltonian $h$ generates the time development mapping $z$ in the variable $t$, the function $w$ generates the mapping $\underset{\sim}{Z} \underset{\sim}{z}, t, \theta)$ in the variable $\theta$. Foillowing this analogy, we introduce the canonical transformation $T$ obtained from the mapping $Z$, just as $M$ came from $\frac{Z}{Z}$. From (2.2.8) we see that $T$ must satisfy

$$
\frac{\partial T}{\partial \theta}=-T L_{w}
$$


We also note that $\mathrm{T}^{-1}$ must satisfy

$$
\frac{\partial T^{-1}}{\partial \theta}=L_{w} T^{-1}
$$

which is analogous to (2.2.11). Finally, any function $f$ satisfying.

$$
\frac{\partial f}{\partial \theta}+\{f, w\}=g
$$

can be solved using

$$
f(\theta)=T^{-1}(\theta) \int_{\theta_{0}}^{\theta} d \theta^{\prime} T\left(\theta^{\prime}\right) g\left(\theta^{\prime}\right)+T^{-1}(\theta) T\left(\theta_{0}\right) f\left(\theta_{0}\right)
$$

which is analogous to $(2 \cdot 2 \cdot 15)$.

These equations are meaningful only if we can prove the existence of $w$. To do this we consider the vector $\mathbf{y}$ given by

$$
v_{j}=-\left.\sum_{i} \gamma_{j k} \frac{\partial z_{k}}{\partial \theta}\right|_{\sim_{\sim}^{-1}(\underset{\sim}{z}, t, \theta), t, \theta}
$$

Suppose we can show that $\mathbf{v}_{\mathbf{j}}$ is the gradient of a function $w$,

$$
v_{j}=\frac{\partial w}{\partial z_{j}}=-\left.\sum_{i}^{\gamma_{j k}} \frac{\partial z_{k}}{\partial \theta}\right|_{\left.z^{-i} \underset{\sim}{z}, t, \theta\right), t, \theta}
$$

Then, by multiplying $(3.1 .8)$ by $\gamma_{i j}$, summing over $j$, and using $(2.1 .3)$ we get

$$
\left.\frac{\partial z_{i}}{\partial \theta}\right|_{z^{-1}(z, t, \theta), t, \theta}=\sum_{j} \gamma_{i j} \frac{\partial w}{\partial z}{ }_{j} .
$$

Transforming the variable in (3.1:9) gives (3.1.1). We see that we must prove that $\mathrm{V}$ is a gradient of a function $w$. Of course, knowing that $\mathrm{y}$ is a gradient, we can find wery simply by integration:

$$
\mathrm{w}=\int^{z} \sum_{\mathrm{m}} \mathrm{v}_{\mathrm{m}} \cdot \mathrm{dz} \mathrm{m}
$$


To prove that $\mathfrak{v}$ is a gradient, we will show that the integrability condition holds. This amounts to showing that the quantity

$$
\frac{\partial v_{1}}{\partial z_{j}}=-\left.\sum_{k m} \gamma_{1 k} \frac{\partial^{2} z_{k}}{\partial z_{m}^{\partial \theta}}\right|_{z^{-1}(z, t, \theta), t, \theta} \cdot \frac{\partial z^{-1} m}{\partial z_{j}}
$$

is symetric in $i$ and $j$. This proof follows from some special properties of the derivative matrix of a canonical transformation. This proof is algebraically tedious, and one need not follow it to understand the rest of this paper, so the reader may choose to skip the rest of this section.

One property which we wish to show is that the derivative matrix of the inverse mapping $\left(\partial z_{i}^{-1} / \partial z_{j}\right)$ is simply related to the derivative matrix of the forward mapping $\left(\partial z_{i} / \partial z_{j}\right)$. By differentiating the following identity,

$$
z_{i}=z_{i}\left(z^{-1}(z, t, \theta), t, \theta\right) \text {, }
$$

it follows that the two matrices are inverses:

$$
\delta_{i k}=\left.\sum_{j} \frac{\partial z_{i}}{\partial z_{j}}\right|_{z^{-1}(z, t, \theta), t, \theta} \cdot \frac{\partial z^{-1} j}{\partial z_{k}} .
$$

However, we also note that by multiplying $(2.1 .6)$ by $\gamma_{j k}$ and summing over $j$ we get

$$
\delta_{i k}=\sum_{j} \frac{\partial z_{i}}{\partial z_{j}}\left(-\sum_{\ell m} \gamma_{j \ell} \frac{\partial z_{m}}{\partial z_{\ell}} \gamma_{m k}\right) .
$$

Comparing (3.1.13) to $(3.1 .14)$, we see (since $\underset{\sim}{z}(\underset{\sim}{z}, t, \theta)$ is invertible) that

$$
\frac{\partial z^{-1} j}{\partial z_{k}}=-\left.\sum_{\ell m} \gamma_{j l} \frac{\partial z_{m}}{\partial z_{\ell}}\right|_{z^{-1}(z, t, \theta), t, \theta} \gamma_{\text {mik }},
$$


relating the inverse mapping derivative matrix to the forward mapping derivative matrix.

From the Poisson bracket relations $(2.1 .6)$ we can prove the other Identity needed to show the symetry of (3.1.11). By differentiating (2.1.6) with respect to $\theta$ we get

$$
\sum \frac{\partial^{2} z_{k}}{\partial \theta \partial z_{m}} \gamma_{m \ell} \frac{\partial z_{n}}{\partial z_{\ell}}=-\sum_{m \ell} \frac{\partial z_{k}}{\partial z_{m}} \gamma_{m \ell} \frac{\partial^{2} z_{n}}{\partial \theta \partial z_{\ell}} .
$$

Now we use (3.1.16) and (3.1.15) to prove the symmetry of (3.1.11). We first insert (3.1.15) into (3.1.11) obtaining

$$
\frac{\partial v_{i}}{\partial z_{j}}=\sum_{k \ell m n}-\left(\gamma_{i k} \frac{\partial^{2} z}{\partial \theta \partial z_{\ell}} \cdot \gamma_{\ell m} \frac{\partial z_{n}}{\partial z_{m}} \gamma_{n j}\right)
$$

Then we use (3.1.16) to put this in the form

$$
\frac{\partial v_{i}}{\partial z_{j}}=\left.\sum_{k \ell m n}\left(\gamma_{i k} \frac{\partial z_{k}}{\partial z_{\ell}} \gamma_{\ell m} \frac{\partial^{2} z_{n}}{\partial z_{m}^{\partial \theta}} \gamma_{n j}\right)\right|_{z_{\sim}^{-1}(z, t, \theta), t, \theta}
$$

By rearranging the dummy indices and using the antisymmetry property of $\gamma_{i j}$, we get

$$
\frac{\partial v_{i}}{\partial z_{j}}=\sum_{k \ell m n}-\left.\left(\gamma_{j k} \frac{\partial^{2} z_{n}}{\partial z_{\ell} \partial \theta} \gamma_{\ell m} \frac{\partial z_{n}}{\partial z_{m}} \gamma_{n i}\right)\right|_{z_{(\underset{\sim}{z}, t, \theta), t, \theta}^{-1} .}
$$

Comparing this to $(3.1 .17)$ we see that the integrability condition holds, and so w does exist. 


\subsection{The New Hamiltonian}

The reason we introduce transformation theory is that we hope to be able to transform to a new system where the Hamiltonian has a simpler form. Upon solving the equations in the simpler system, we can transform back to obtain the solutions in the original system.

The new Hamiltonian $\mathrm{K}$ must have the property of giving the equations of motion for the transformed variables $\underset{\sim}{Z}(\underset{\sim}{z}, t, \theta)$ :

$$
\dot{z}_{i}=\left\{z_{i}, k(\underset{\sim}{z}(z, t, \theta), t, \theta)\right\} \text {. }
$$

In this equation, overdot refers to the time derivative along a trajectory. Using the old Hamiltonian, we can also find $z_{1}$ :

$$
\dot{z}_{i}=\frac{\partial z_{i}}{\partial t}+\left\{z_{i}, h(z, t)\right\} .
$$

Introducing the function $\left.H(\underset{\sim}{z}, t, \theta)=h_{i}{\underset{\sim}{z}}^{-1}(\underset{\sim}{z}, t, \theta), t\right)$, we can write $(3.2 .2)$ in the form

$$
\left.\dot{z}_{1}=\left\{z_{i}, H(\underset{\sim}{z}(\underset{z}{z}, t, \theta), t, \theta)\right)\right\}+\frac{\partial z_{i}}{\partial t} .
$$

Hence we need only' find a function $R$ such that

$$
\frac{\partial z_{i}}{\partial t}=\left\{z_{i}, R(\underset{\sim}{z}(\underset{\sim}{z}, t, \theta), t, \theta)\right\},
$$

than the new Hamiltonian $\mathrm{K}$ is given by $\mathrm{K}=\mathrm{H}+\mathrm{R}$.

That such an $R$ exists is evident from the following considerations. $\underset{\sim}{Z}(\underset{\sim}{z}, t, \theta)$ is a canonical transformation for all $t$ and $\theta$. Since it is canonical for all $\theta$, we were able to conclude that w exists satisfying (3.1.1). Likewise, since $\underset{\sim}{Z}(\underset{\sim}{z}, t ; \theta)$ is canonical for all $t$, we conclude that an $R$ exists satisfylng (3.2.4). Knowing that $R$ exists, we can be more secure about finding it.

To find $R$, we consider equations (3.1.1) and (3.2.4) written in operator form. 


$$
\begin{aligned}
& \frac{\partial T}{\partial \theta}=-T L_{w} \\
& \frac{\partial T}{\partial t}=-T L_{R}
\end{aligned}
$$

Now we equate the second partials of $T$ calculated from the two above equations.

$$
\frac{\partial T}{\partial t} L_{w}+T L_{\frac{\partial w}{\partial t}}=\frac{\partial T}{\partial \theta} L_{R}+T L_{\frac{\partial R}{\partial \theta}}
$$

By using $(3.2 .5)$ and $(3.2 .6)$ again we get

$$
L_{\frac{\partial R}{\partial \theta}}+L_{\{R, w\}}=L_{\frac{\partial w}{\partial t}} .
$$

From equation $(3.2 .8)$ we would like to deduce the relationship between $R$ and $w$. As discussed in section 2.1 , we can only deduce

$$
\frac{\partial R}{\partial \theta}+\{R, w\}=\frac{\partial w}{\partial t}+g,
$$

where $g$ is any function independent of $\underset{\sim}{z}$. However, we can set $g$ equal to zero here, since $R$ has relevance only through its Poisson bracket relations as In (3.2.4). The addition of a function independent of $z$ to $R$ does not affect any Poisson bracket relations. Setting $g=0$, we get

$$
\frac{\partial R}{\partial \theta}+\{R, w\}=\frac{\partial w}{\partial t}
$$

Following the discussion in the last section, this equation can be integrated using (3.1.6). We use $\theta_{0}=0$ since then $T$ reduces to the identity implying that $R\left(\theta_{0}=0\right)$ vanishes. This gives the result for $R$ :

$$
R(\theta)=T^{-1}(\theta) \int_{0}^{\theta} d \theta^{\prime} T\left(\theta^{\prime}\right) \frac{\partial w}{\partial t}\left(\theta^{\prime}\right) .
$$

If we now use $K=H+R$, we get the new Hamiltonian.

$$
K(\theta)=T^{-1}(\theta) h+T^{-1}(\theta) \int_{0}^{\theta} d \theta^{\prime} T\left(\theta^{\prime}\right) \frac{\partial w}{\partial t}\left(\theta^{\prime}\right) .
$$


This result was first obtalned by Dewar [10], but with different conventions.

To compare (3.2.12) with more familiar equations, we consider the case where the transformation is time independent. In this case $\frac{\partial w}{\partial t}=0$, so

$$
K(\theta)=T^{-1}(\theta) h \text {. }
$$

Using the definition of $\mathrm{T}$, this can be written

$$
x(z(z, t, \theta), t, \theta)=h(z, t, \theta),
$$

which simply says that the new Hamiltonian evaluated at the mapped positions equals the old Hamiltonian evaluated at the old positions.

In the time dependent case, the remainder function $R$ must equal $\partial F / \partial t$, the time derivative of the generating function of mixed variables, which appears in Hamilton-Jacobl theory. Using this fact, one can derive the relation between $w$ and $F$. The relation between $w$ and $F$ was first found by Dewar by $a$ different method [11].

A final point which we wish to discuss is the transformation of the Liouville equation. If $f$ is the old density and $F$ (not the generating function) Is the new density, they must be related by

$$
\dot{F}=\mathrm{T}^{-1} \mathbf{F} \text {, }
$$

since the new density at the new point equals the old density at the old point. Since f satisfies

$$
\frac{\partial f}{\partial t}+\{f, h\}=0,
$$

we can show that $F$ satisfies

$$
\frac{\partial F}{\partial t}+\{F, K\}=0,
$$

as it must if $\mathrm{F}$ is the density in the transformed system. One shows this by combining (3.2.6) and (3.2.12) to obtain

$$
\frac{\partial T}{\partial t}=-T L_{K}+L_{h} T \text {, }
$$

and using this expression in differentiating $f=\dot{T F}$ with respect to time. 


\section{3:3 An Illustration}

To illustrate this theory, we consider a specific transformation. We consider the transformation given by the generating function

$$
w=-\theta t p^{2} \text {. }
$$

We must first find the variable mapping $Q(q, p, t, \theta), P(q, p, t, \theta)$. This mapping is found by solving (3.1.1), which becomes in this case

$$
\frac{\partial Q}{\partial \theta}=-2 \theta t P
$$

and

$$
\frac{\partial P}{\partial \theta}=0
$$

These equations are trivially solved giving

$$
\begin{aligned}
& Q=q-\theta^{2} t P \\
& P=p
\end{aligned}
$$

We note that the boundary conditions (3.1.2) have been satisfied.

The operator $T$ corresponding to (3.3.3) acts on a function $f$ as follows. If $\operatorname{Tf}=g$, then

$$
g(q, p ; t, \theta)=f\left(q-\theta^{2} t p, p, t, \theta\right) \text {. }
$$

We could also use the operator notation to deduce (3.3.4). The Lie operator in this case is given by

$$
L_{w}=+2 \theta t p \frac{\partial}{\partial q} \text {. }
$$

The equation for $\mathrm{T}$ is

$$
\frac{\partial T}{\partial \theta}=-T 2 \theta t p \frac{\partial}{\partial q}
$$

This equation is trivially solved giving

$$
\begin{aligned}
T & =\exp \left(-\theta^{2} \operatorname{tp} \frac{\partial}{\partial q}\right) \\
& =\sum_{n=0}^{\infty} \frac{1}{n !}\left(-\theta^{2} t p\right)^{n}\left(\frac{\partial}{\partial q}\right)^{n},
\end{aligned}
$$


and this is just the Taylor series expression for the shift operator in $(3.3 .4)$

We now calculate the new Hamiltonian using (3.2.12) in the case where the original system is a free particle system, $h=p^{2} / 2 m$. In this case we see that $T^{-1} h=h$ and $T \frac{\partial w}{\partial t}=\frac{\partial w}{\partial t}$, so that $K$ is easily calculated giving

$$
K=\frac{1}{2 m} p^{2}-\frac{1}{2} \theta^{2} p^{2}
$$

We see that the motion in the new system is free particle motion with the new mass given by

$$
M(\theta)=\frac{m}{1-\theta^{2} m} .
$$

We see that we can transform away the Hamiltonian entirely in this simple case, by ehoosing $\theta^{2}=\frac{1}{m}$. In this new system $q$ and $p$ are constant. To find the motion in the original system, we first apply the inverse transformation

$$
\begin{aligned}
& \mathrm{Q}^{-1}=\mathrm{q}+\mathrm{pt} / \mathrm{m} . \\
& \mathrm{P}^{-1}=\mathrm{p}
\end{aligned}
$$

Then we substitute the trajectories in the transformed system $q_{K}(t)=q_{0}$ and $f_{K}(t)=P_{0}$ to get the trajectories in the original system

$$
\begin{aligned}
& g_{h}(t)=q_{K}(t)+\frac{1}{m} t f_{K}(t)=q_{0}+\frac{1}{m} t p_{0} \\
& g_{h}(t)=f_{K}(t)=p_{0}
\end{aligned}
$$




\subsection{The Symmetry-Equals-Invariant Theorem (Noether's theorem)}

Noether's theorem (ref. [1] p. 83) in Lagrangian dynamics states that 17 otis given a family of transformations which leaves the form of the Lagranglan unchanged except for the addition of a total time derivative, then one can construct an invariant of the motion. Conversely, given an invariant of the motion, one can construct a family of transformations which leave the Lagrangian unchanged except for the addition of a total time derivative.

Since there is a direct correlation between Lagrangian dynamics and Hamiltonian dynamics, one expects a similar theorem to hold in Hamiltonian mechanics. Th1s theorem has in fact been discussed by Whittaker [2] and Anderson [15]. The advantage of the Hamiltonian formulation is that the relevant transformations are the time dependent canonical transformations, the natural transformations which occur in Hamiltonian mechanics. In contrast, the Lagrangian theorem requires the use of path transformations (see ref. [1] p. 83) not just time dependent coordinate transformations.

However, one drawback of the theorems presented in refs. [2] and [15] is that they consider only infinitesimal transformations. With the finite transformation theory presented earlier, this can be remedied, as was done in ref. [16]. Here we present this theorem using the operator notation of this paper.

Given a system which evolves according to a Hamiltonian $h$, we say that $h$ has a symetry if there exists a transformation family $T(\theta)$ which leaves the Hamiltonian unchanged up to the addition of a phase space independent function:

where

$$
k=h+f
$$

$$
\frac{\partial f}{\partial z_{1}}=0 \text { for all } 1
$$


The reason we allow. the addition of $f$ is that it will not affect the motion in the new system, since its Poisson bracket with any function vanishes.

We define an invariant to be any function $g$ whose total time derivative along a trajectory vanishes:

$$
\frac{\partial g}{\partial t}+\{g ; h\}=0
$$

We will prove that given a symmetry, one can construct an invariant, and given an invariant, one can construct a symetry.

First we suppose that we are given a symetry. Using (3.4.1) in (3.2.12), we have

$$
\mathrm{h}+\mathbf{f}=\mathrm{T}^{-1}(\theta) \mathrm{h}+\mathrm{T}^{-1}(\theta) \int_{0}^{\theta} \mathrm{d} \theta^{\prime} \mathrm{T}\left(\theta^{\prime}\right) \frac{\partial \mathrm{w}}{\partial \mathrm{t}}\left(\theta^{\prime}\right)
$$

where $\mathrm{w}$ is constructed from $\mathrm{T}$ as discussed in 3.1 . We premultiply this equation by $T$, and note that $T f=f$, since $f$ does not depend on the phase space variables.

$$
T(\theta) h+f=h+\int_{0}^{\theta} d \theta^{\prime} T\left(\theta^{\prime}\right) \frac{\partial w}{\partial t}\left(\theta^{\prime}\right)
$$

Differentiating (3.4.5) with respect to $\theta$; and premultiplying by $\mathrm{T}^{-1}$ gives

$$
: \frac{\partial w}{\partial t}-\frac{\partial f}{\partial \theta}+\{w, h\}=0 \text {. }
$$

Thus the function $g(z, t, \theta)=w-\int_{d \tau}^{t} \partial f / \partial \theta$ is an invariant for alle.

To prove the other half of the theorem, we assume we have an invariant g - From this invariant we construct a transformation $T(\theta)$ using $w=g$. The new Hamiltonian $K$ is then given by

$$
K(\theta)=T^{-1}(\theta) h+T^{-1}(\theta) \int_{0}^{\theta} d \theta^{\prime} T\left(\theta^{\prime}\right) \frac{\partial g}{\partial t} .
$$

Premultiplying $\mathrm{T}$ and differentiating with respect to $\theta$ gives

$$
-T L_{8} K+T \frac{\partial K}{\partial \theta}=T \frac{\partial g}{\partial t}
$$


which Impiles that

$$
\frac{\partial K}{\partial \theta}+\{K, g\}=\frac{\partial g}{\partial t}
$$

We now use the fact that $g$ is an invariant. This gives

$$
\frac{\partial(\mathrm{K}-\mathrm{h})}{\partial \theta}+\{\mathrm{K}-\mathrm{h}, \mathrm{g}\}=0,
$$

since $h$ is independent of $\theta$. We define $b$ by $b=T(K-h)$. In terms of b, (3.4.9) reads

$$
\frac{\partial \mathrm{b}}{\partial \theta}=0
$$

Since $b=0$ at $\theta=0, b$ vanishes for all $\theta$, from which we deduce $k=h$, proving the theorem.

As stated here, the theorem is more powerful than it is in its usual form. Here we find that the generating function $g$ is an invariant for all values of $\theta$, whereas usually only $g$ at $\theta=0$ is shown to be an invariant.

We are used to thinking of the explicitly. time independent invariants such as momentum and angular momentum. This theorem shows that symmetries are associated even with time dependent invariants such as the function

$$
g=p \cos \omega_{0} t+q \sin \omega_{0} t
$$

for the harmonic oscillator $h=\frac{1}{2} \omega_{0}\left(q^{2}+p^{2}\right)$. In general a Hamiltonian in $2 \mathrm{~N}$ dimensional phase space has $2 \mathrm{~N}$ functionally independent invariants, and hence its symetry Lie group is $2 \mathrm{~N}$ dimensional. 
4. Deprit Perturbation. Theory

In this section we show how to construct a perturbation theory from the general transformation theory of the previous chapter. Historically the development was in the opposite direction. Deprit constructed power series representations of the transformation, and later Dewar gave the general representation applicable even when the transformation is not analytic. The basic 1dea of the perturbation theory is that the Hamiltonian which we wish to solve consists of a solvable term plus unsolvable terms which are ordered in a small parameter. We equate this small parameter to the parameter $\theta$ of a Lie transform which is to be determined. We then expand the Lie transform in powers of the parameter $\theta$, and use the expansions in equation (3.2.12). We end up with an equation for each order relating the new Hamiltonian to the transformation and to the old Hamiltonian.

The next, stage in the transformation theory is to pick the transformation, thereby choosing the form of the new Hamiltonian. Ideally we would like to. pick the transformation so that $K$ vanishes in all the higher orders. However, this choice is not always best since it may lead to secular terms or small denominators in the transformation, making it useless for a discussion of long time effects. The scheme we adopt is then to transform away as many terms as possible. The slowly varying terms, which give rise to secularities and small denominators, must be kept in the new Hamiltonian. This scheme usually simplifies the analysis to some degree since at least the rapidly varying terms are transformed away. Sometimes this scheme in effect solves the problem when the slowly varying terms depend only on the momenta.

The structure of this chapter is as follows. In the first section we derive the power series expressions for the Lie transform theory. Then we illustrate the method of choosing the transformations outlined in the previous 
paragraph by doing two examples. However, it must be noted that the described method of choosing is not the only one. A. nice feature of the Deprit perturbation theory is that one can use any method to select the transformation: The Important thing is to choose the transformation. so: that the new Hamiltonian is as easy to analyze as possible. Finally we .. include a section on superconvergence, showing how it occurs in Lie transform theory. In this section we also discuss the practical usage of the superconvergence technique. 


\subsection{Deprit Perturbation Series Relations}

This perturbation theory relies on the power. series expansions of the Lie transforms. In this section we assume that the Hamiltonian $h$ is ordered In a parameter which we equate to the Lie transform parameter. We then Insert the power series expansions into (3.2.12) to obtain the expression for the $n^{\text {th }}$ order term in the new Hamiltonian.

The starting point is the assumption that the objects.h,w, K, and $\mathrm{T}$ can all be expanded in power series. For $h, K$, and $T$ we have

$$
\begin{aligned}
& \left.h(\underset{\sim}{z}, t, \theta)=\sum_{n=0}^{\infty} \theta^{n_{h}}{ }_{n}^{(z,}, t\right), \\
& K(\underset{\sim}{z}, t, \theta)=\sum_{n=0}^{\infty} \theta^{n_{K_{n}}(\underset{\sim}{z}, t),} \\
& T(t, \theta)=\sum_{n=0}^{\infty} \theta^{n T_{n}(t) .}
\end{aligned}
$$

and

However, for we assume a slightly different form

$$
w(\underset{\sim}{z}, t, \theta)=\sum_{n=0}^{\infty} \theta^{n} w_{n+1}(\underset{\sim}{z}, t) \text {. }
$$

The reason for this choice is that w occurs in (3.2.12) along with an integral over $d \theta$ which effectively boosts the order of $w$ by 1 in all the equations.

We also assume that the transformation is close to the identity. This Is necessary for perturbation theory to work at all. This implies that $T_{0}=I$, putting $\theta=0$. It also implies $\mathrm{K}_{0}=\mathrm{h}_{0}$ since in $(3.2 .12)$ the integral is at least of order $\theta$. 
We first find the form for the operator $T$ to all orders. We start with the relation

$$
\frac{\partial T}{\partial \theta}=-T L_{w} .
$$

To use this relation we will need to know the series for $L_{w}$. Since the map $g \rightarrow \mathrm{L}_{\mathrm{g}}$ is linear, we have

$$
L_{w}=\sum_{n=0}^{\infty} \theta^{n} L_{w_{n+1}}
$$

We abbreviate the operator $L_{w_{n}}$ by $L_{n}$. Now we simply take the series representations for $\mathrm{L}_{\mathrm{.}}$ and $\mathrm{T}$ and insert them into (4.1.5). As a result we calculate the following recursion relation:

$$
T_{n}=\frac{-1}{n} \sum_{m=0}^{n-1} T_{m} L_{n-m} .
$$

By iterating this relation we arrive at

$$
\mathrm{T}_{\mathrm{n}}=\sum_{\substack{\left(\mathrm{m}_{1}, \ldots, \mathrm{m}_{\mathrm{r}}\right) \\ \mathrm{n}>\mathrm{m}_{1}>\mathrm{m}_{2}>\ldots>\mathrm{m}_{\mathrm{r}}>0}}\left(\frac{-1}{\mathrm{n}}\right)\left(\frac{-1}{\mathrm{~m}_{1}}\right) \cdots\left(\frac{-1}{\mathrm{~m}_{\mathrm{r}}}\right) \mathrm{L}_{\mathrm{m}_{\mathrm{r}}} \cdots \mathrm{L}_{\mathrm{m}_{2}-\mathrm{m}_{3}} \mathrm{~L}_{\mathrm{m}_{1}-\mathrm{m}_{2}} \mathrm{~L}_{n-\mathrm{m}_{1}} .
$$

The sum is over all sets of integers $m_{1}, \ldots, m_{r}$ satisfying $n>m_{1}>m_{2}>\ldots>m_{r}>0$.

This expression was first derived by Littlejohn [17].

From the expression $\frac{\partial \mathrm{T}^{-1}}{\partial \theta}=\mathrm{L}_{\mathrm{w}} \mathrm{T}^{-1}$, we can derive similar relations for $\mathrm{T}^{-1}$ The results are

$$
\mathrm{T}_{\mathrm{n}}^{-1}=\frac{1}{\mathrm{n}} \sum_{m=0}^{\mathrm{n}-1} \mathrm{~L}_{\mathrm{n}-\mathrm{m}} \mathrm{T}_{\mathrm{m}}^{-1}
$$

which gives

$$
\mathrm{T}_{\mathrm{n}}^{-1}=\sum_{\substack{\left(\mathrm{m}_{1}, \ldots, \mathrm{m}_{\mathrm{r}}\right) \\ \mathrm{n}>\mathrm{m}_{1}>\mathrm{m}_{2}>\ldots>\mathrm{m}_{\mathrm{r}}>0}}\left(\frac{1}{\mathrm{n}}\right)\left(\frac{1}{\mathrm{~m}_{1}}\right) \cdots\left(\frac{1}{\mathrm{~m}_{\mathrm{r}}}\right) \mathrm{L}_{\mathrm{n}-\mathrm{m}_{1}} \mathrm{~L}_{\mathrm{m}_{1}-\mathrm{m}_{2}} \cdots \mathrm{L}_{\mathrm{m}_{\mathrm{r}}} .
$$

To find the expression for $K$, we could insert the series for $T, w$, and $h$ into (3.2.12). If this were done, it would be better to choose the conventions of ref. [16]. However, by manipulating (3.2..12), one can derive the power series 
for $\mathrm{K}$ from the resulting equation [17]. If one finds $\mathrm{K}$ by this method it is better to usc the conventions given here, as discussed in Ch. 5 .

Premultiplying $(3.2 .12)$ by $T$ and differentiation with respect to

$\theta$ gives

$$
\frac{\partial T}{\partial \theta} K+T \frac{\partial K}{\partial \theta}=\frac{\partial h}{\partial \theta}+T \frac{\partial w}{\partial t}
$$

Using (3.2.5), and premultiplying by $\mathrm{T}^{-1}$ then gives

$$
\frac{\partial w}{\partial t}=\frac{\partial K}{\partial \theta}-I_{w} K-T^{-1} \frac{\partial h}{\partial \theta}
$$

Inserting the series expressions for $K, T$, and $h$ gives (in $n^{\text {th }}$ order)

$$
\frac{\partial w_{n}}{\partial t}=n k_{n}-\sum_{m=0}^{n-1} L_{n-m} K_{m}-\sum_{m=1}^{n} T_{n-m}^{-1} m h_{m} \text {. }
$$

By writing out the first term of the first sum, and the last term of the sum we get the final answer (note $\mathrm{K}_{0}=\mathrm{h}_{0}$ ):

$$
\frac{\partial w_{n}}{\partial t}+\left\{w_{n}, h_{o}\right\}=n k_{n}-n h_{n}-\sum_{m=1}^{n \cdot n \cdot 1}\left(L_{n-m} k_{m}+m T_{n-m}^{-1} h_{m}\right)
$$

This is the form with which we work when doing $n^{\text {th }}$ order perturbation theory. After calculating through order $(n-1)$, we know all of the quantities in the sum in the above equation. We also know $h_{n}$. We then pick $K_{n}$ in a manner described in the next section. Finally we find $w_{n}$ by integrating the right side of (4.1.14) along a trajectory. At this point all of the quantities are known through order $n$, hence we know the motion through $n^{\text {th }}$ order, and we can begin the order $(n+1)$ calculation.

For future reference, we give the formulas for the various quantities through fourth order. 


$$
\begin{aligned}
& T_{0}=I \\
& T_{1}=-L_{1} \\
& T_{2}=-\frac{1}{2} L_{2}+\frac{1}{2} L_{1}^{2} \\
& T_{3}=-\frac{1}{3} L_{3}+\frac{1}{6} L_{2} L_{1}+\frac{1}{3} L_{1} L_{2}-\frac{1}{6} L_{1}^{3} \\
& T_{4}=-\frac{1}{4} L_{4}+\frac{1}{12} L_{3} L_{1}+\frac{1}{8} L_{2}^{2}+\frac{1}{4} L_{1} L_{3} \\
& T_{0}^{-1}=\frac{1}{24} L_{2} L_{1}^{2}-\frac{1}{12} L_{1} L_{2} L_{1}-\frac{1}{8} L_{1}^{2} L_{2}+\frac{1}{24} L_{1}^{4} \\
& T_{1}^{-1}=L_{1} \\
& T_{2}^{-1}=\frac{1}{2} L_{2}+\frac{1}{2} L_{1}^{2} \\
& T_{3}^{-1}=\frac{1}{3} L_{3}+\frac{1}{6} L_{1} L_{2}+\frac{1}{3} L_{2} L_{1}+\frac{1}{6} L_{1}^{3} \\
& T_{4}^{-1}=\frac{1}{4} L_{4}+\frac{1}{12} L_{1} L_{3}+\frac{1}{8} L_{2}^{2}+\frac{1}{4} L_{3} L_{1} \\
& T_{1}
\end{aligned}
$$

We note that $T_{n}^{-1}=T_{n}^{+}$, where the dagger indicates hermitian conjugate. To find the hermitian conjugate of a product of operators, we reverse the order of the product, and then take the hermitian conjugate of each of the multipliers :

$$
(\mathrm{AB})^{+}={\mathrm{B}-\mathrm{A}^{+}}^{+}
$$

Since $L_{n}^{+}=-L_{n}\left(L_{n}\right.$ is antihermitian), we see that $T_{n}^{-1}=T_{n}^{+}$is true in (4.1.16). This fact is proven in general by noting that the differential equations for $\mathrm{T}$ and $\mathrm{T}^{-1}$ are hermitian conjugates. 
The equations for $\mathrm{K}_{\mathrm{n}}$ to fourth order are:

$$
\mathrm{K}_{0}=\mathrm{h}_{\mathrm{o}}
$$

$$
\frac{\partial w_{1}}{\partial t}+\left\{w_{1}, h_{0}\right\}=k_{1}-h_{1}
$$

$$
\frac{\partial w_{2}}{\partial t}+\left\{w_{2}, h_{0}\right\}=2\left(k_{2}-h_{2}\right)-L_{1}\left(k_{1}+h_{1}\right)
$$

$$
\begin{aligned}
& \frac{\partial w_{j}}{\partial t}+\left\{w_{3}, h_{0}\right\}=3\left(k_{3}-h_{3}\right)-L_{1}\left(K_{2}+2 h_{2}\right)-L_{2}\left(K_{1}+\frac{1}{2} h_{1}\right)-\frac{1}{2} L_{1}^{2} h_{1} \\
& \frac{\partial w_{4}}{\partial t}+\left\{w_{4}, h_{0}\right\}=4\left(K_{4}-h_{4}\right)-L_{1}\left(K_{3}+3 h_{3}\right)-L_{2}\left(K_{2}+h_{2}\right)-L_{1}{ }^{2} h_{2} \\
& -L_{3}\left(K_{1}+\frac{1}{3} h_{1}\right)-\frac{1}{6}\left(L_{1} L_{2}+2 L_{2} L_{1}+L_{1}^{3}\right) h_{1} \text {. }
\end{aligned}
$$

In passing, we note that Littlejohn [17] has written a computer program to find these equations to arbitrary order. 


\subsection{The Anharmonic Oscillator}

In this section we apply the Deprit perturbation technique to a sample problem as an illustration. The problem we consider is that of a particle moving in a potential with cubic and fifth power force nonlinearities. The Hamiltonian in this case is given by

$$
h=\frac{1}{2} p^{2}+\frac{1}{2} w_{o}^{2} q^{2}+\frac{\varepsilon}{4} w_{o}^{2} q^{4}+\frac{\varepsilon^{2}}{8} a w_{o}^{3} q^{6}
$$

We solve for the motion near the bottom of the well where the nonlinearity is small.

The unperturbed Hamiltonian is a simple harmonic oscillator. For converience we use the (unperturbed) action angle variables defined by

$$
\begin{aligned}
& p=-\sqrt{2 \omega j} \sin \phi \\
& q=\sqrt{2 j / \omega_{o}} \cos \phi
\end{aligned}
$$

In terms of these variables the Hamiltonian takes the form

$$
h=\omega_{o} j+\varepsilon j^{2} \cos ^{4} \phi+\varepsilon^{2} a j^{3} \cos ^{6} \phi .
$$

The ordering scheme for this Hamiltonian $h$ is of course

$$
\begin{aligned}
& h_{0}=\omega_{0} j \\
& h_{1}=j^{2} \cos ^{4} \phi \\
& h_{2}=a j^{3} \cos ^{6} \phi \\
& h_{n}=0 \text { for } n>2
\end{aligned}
$$

We equate the Lie transform parameter $\theta$ to the small parameter $\varepsilon$.

The first step in perturbation theory is to solve the unperturbed problem; i:e. we must find the time development operator. In this case the time development mapping is

$$
\begin{aligned}
& \varphi(\phi, j, t)=\phi+\omega_{0} t \\
& f(\phi, j, t)=j .
\end{aligned}
$$


The time development mapping $M_{0}$ is therefore given by

$$
f=M_{0} g \Rightarrow f(\phi, j, t)=g\left(\phi+w_{0} t, j, t\right) \text {. }
$$

To do first order perturbation theory we consider the first order Deprit equation $(4.1 .17 \mathrm{~b})$. In this case we have

$$
\begin{aligned}
\frac{\partial w_{1}}{\partial t}+\left\{w_{1}, h_{0}\right\} & =K_{1}(\phi, j, t)-j^{2} \cos ^{4} \phi, \\
& =k_{1}-j^{2}\left(\frac{3}{8}+\frac{1}{2} \cos 2 \phi+\frac{1}{8} \cos 4 \phi\right) .
\end{aligned}
$$

We can solve for $w_{1}$ using (2.2.20). Since we are looking for any solution to $(4.2 .7)$, we use the indefinite integral:

$$
\begin{gathered}
w_{1}=-\int^{t} d \tau\left[j^{2}\left(\frac{3}{8}+\frac{1}{2} \cos \left[2 \phi+\omega_{0}(\tau-t)\right]+\frac{1}{8} \cos \left[4 \phi+4 \omega_{0}(\tau-t)\right]\right)\right. \\
\left.-k_{1}\left(j, \phi+\omega_{0}(\tau-t), \tau\right)\right]
\end{gathered}
$$

We would like to pick $\mathrm{K}_{1}=0$, but since $\mathrm{h}_{1}$ has a nonzero time average this would cause $w_{1}$ to be secular (unbounded in time). Therefore we pick $k_{1}$ to be the time average of $h_{1}$ along a particle orbit:

$$
\begin{gathered}
k_{1}(j)=\frac{\omega_{0}}{2 \pi} \int_{0}^{2 \pi / \omega_{0}} d \tau h_{1}^{-}\left(j, \phi+\omega_{0} \tau\right) \\
=\frac{3}{8} j^{2} .
\end{gathered}
$$

Inserting this result into $(4.2 .8)$ gives

$$
w_{1}=-j^{2}\left(\frac{1}{32} \sin 4 \phi+\frac{1}{4} \sin 2 \phi\right) / \omega_{0} .
$$

We have completed our calculation to first order since we have $w_{1}$ and $k_{1}$. Introducing the transformed variables $\Phi$ and $J$ we know that they evolve in time according to

$$
\begin{aligned}
& J=J_{0} \\
& \Phi=\Phi_{0}+\left(\omega_{0}+\frac{3}{4} \varepsilon J_{0}\right) t,
\end{aligned}
$$


since to first order the transformed Hamiltonian is

$$
K(J)=\omega_{0} J+\frac{3}{8} \varepsilon J^{2}
$$

Also, by applying the inverse transform (4.1.16) we know that the old and new variables are related by $z_{i}=z_{i}+\left\{w_{1}, z_{i}\right\}$ giving

$$
\begin{aligned}
& \phi=\Phi+\varepsilon J\left(\frac{1}{16} \sin 4 \Phi+\frac{1}{2} \sin 2 \Phi\right) / \omega_{0} \\
& j=J-\varepsilon J^{2}\left(\frac{1}{8} \cos 4 \Phi+\frac{1}{2} \cos 2 \Phi\right) / \omega_{\circ} .
\end{aligned}
$$

Inserting the time development of $\mathrm{J}$ and $\Phi(4.2 .11)$ and (4.2.12) into (4.2.14) and (4.2.15) gives the time development of $j$ and $\phi$.

Going to second order, we insert our results for $k_{1}$ and $w_{1}$ into $(4.1 .17 \mathrm{c})$. The result is

$$
\begin{aligned}
\frac{\partial w_{2}}{\partial t}+\left\{w_{2}, h_{0}\right\}=2\left[k_{2}\right. & -\frac{a j^{3}}{32}(10+15 \cos 2 \phi+6 \cos 4 \phi+\cos 6 \phi) \\
& \left.+\frac{j^{3}}{64 \omega_{0}}(17+33 \cos 2 \phi+6 \cos 4 \phi-2 \cos 6 \phi)\right] .
\end{aligned}
$$

Since the average along a particle orbit is essentially a phase average, we can pick off the terms in $\mathrm{K}_{2}$ by inspection.

$$
K_{2}(j)=\left(\frac{5 a}{16}-\frac{17}{64 \omega_{0}}\right) j^{3}
$$

We could now find $w_{2}$ from $(4.2 .16)$ and proceed to third order.

To understand the physical meaning of this transformation, we note that these transformations do not change the topology of the variables. $\Phi$ and $\phi$ are both defined modulo $2 \pi$. Hence we can calculate the action I of the oscillator

$$
I=\frac{1}{2 \pi} \oint p(q) d q=\frac{1}{2 \pi} \int_{0}^{2 \pi} J(\Phi) d \Phi
$$


Since $J$ is constant along an orbit, it follows that the action is $J$. The transformation $\mathrm{T}$ takes the action angle variables of the unperturbed oscillator to the action angle variables of the perturbed system.

The physical relationship which one usually desires for an oscillator is the frequency versus energy function. The numerical value of the Hamiltonian is unchanged in a time independent transformation, so we can get this relationship by eliminating $J$ from the expressions for the new Hamiltonian and the frequency:

$$
\begin{aligned}
& K=\omega_{0} J+\frac{3}{8} \varepsilon J^{2}+\varepsilon^{2}\left(\frac{5 a}{16}-\frac{17}{64 \omega}\right) J^{3} \\
& \omega=\omega_{0}+\frac{3}{4} \varepsilon J+\varepsilon^{2}\left(\frac{15 a}{16}-\frac{5 i}{64 \omega_{0}}\right) J^{2} .
\end{aligned}
$$

To second order in $\varepsilon$ this result is

$$
\omega=\omega_{0}+\frac{3}{4} \varepsilon\left(\frac{E}{\omega_{0}}\right)+\varepsilon^{2}\left(\frac{E}{w_{0}}\right)^{2}\left(\frac{15 a}{16}-\frac{69}{640_{0}}\right)
$$

We have set $\mathrm{K}=\mathrm{E}$.

We now compare our results to a known solvable system, a pendulum.

In this case the Hamiltonian is

$$
\begin{gathered}
h=\frac{1}{2} p^{2}+\omega_{0}^{2}(1-\cos q) \\
=\frac{1}{2} p^{2}+\frac{1}{2} \omega_{0}^{2} q^{2}-\frac{1}{4 !} \omega_{0}^{2} q^{4}+\frac{1}{6 !} \omega_{0}^{2} q^{6} .
\end{gathered}
$$

Comparing with (4.2.1), we see that $\varepsilon=-\frac{1}{6}$ and $a=\frac{2}{5 \omega_{0}}$. Perturbation theory thus gives

$$
\omega=\omega_{0}\left(1-\frac{1}{8} \frac{E}{\omega_{0}^{2}}-\frac{5}{256}\left(\frac{E}{\omega_{0}^{2}}\right)^{2}\right)
$$

The exact result (ref. [18], p. 112) is

$$
\omega=\omega_{0}\left[2 K\left(\sqrt{\frac{E}{2 \omega_{0}^{2}}}\right)\right]^{-1} \text {, }
$$

which yields (4.2.24) upon expansion ( $K$ is the complete elliptic integrai).

A point to note in this example is that we generate the exact solution as a power series in $\varepsilon$. It could happen that there are singularities a $\varepsilon=0$, e.g. $\omega(\varepsilon)$ In $(4.2 .20)$ could contain a term like $e^{-1 / \varepsilon^{2}}$. Perturbation theory 
cannot pick these terms up since analyticity in $\varepsilon$ is assumed from the outset. But, if the exact solution is a power series, we should be able to obtain it term by term using perturbation theory. 


\subsection{The Parametrically Driven Anharmonic Oscillator}

We now show what happens when we add a time dependent perturbation to the problem of the previous section. The particular perturbation we choose is a parametric time dependence, so that $h$ has the form

$$
h=\frac{1}{2} p^{2}+\frac{1}{2} \omega_{0}^{2}(1+\alpha \sin \Omega t) q^{2}+\frac{\varepsilon}{4} \omega_{0}^{2} q^{4}
$$

Before embarking on this problem, we discuss the ordering scheme. If we wish to be careful, in any calculation we must assign an order to each of the various terms in a Hamiltonian. Then we can approach the question of errors. However, in most work in physics only very rough error estimates are given. Secondly, a națural small parameter may not appear obvious. For example, in the problem of the last section we could have set $\varepsilon=1$ and followed the same perturbation calculation. The Deprit equations would keep track of the orders, and we would have to keep in mind that our solution is good only when the term we treat as a perturbation is small. In the previous calculation this would mean small energy. Hence in doing a calculation it is not necessary that $h_{2}$ have an $\varepsilon^{2}$ in front of it, instead we know that upon doing a calculation to first order, errors of the size of $h_{2}$ remain.

In the above Hamiltonian we have two small parameters, $\alpha$ and $\varepsilon$. We want to solve it in the case where the time dependent perturbation is smaller than the nonlinearity, so we choose

$$
\begin{aligned}
& h_{0}=\frac{1}{2} p^{2}+\frac{1}{2} \omega_{0}^{2} q^{2} . \\
& h_{1}=\frac{\varepsilon}{4} \omega_{0}^{2} q^{4} \\
& h_{2}=\frac{1}{2} \alpha \omega_{0}^{2} \sin \Omega t q^{2} \\
& h_{n}=0 \text { for } n>0 .
\end{aligned}
$$


In doing this calculation we set $\theta=1$, letting the Deprit perturbation equations keep track of the ordering.

We again switch to the more convenient variables. We have done the first order analysis in the previous section, and so we know $h_{1}$ and $k_{1}$. Here we start with $h_{2}$ (and the more convenient variables).

$$
h_{2}=\frac{\alpha \omega_{0} j}{2}\left[\sin \Omega t+\frac{1}{2} \sin (\Omega t+2 \phi)+\frac{1}{2} \sin (5 i t-2 \phi)\right]
$$

This term modifies $(4.2 .16)$ to be

$$
\begin{aligned}
\frac{\partial w_{2}}{\partial t}+\left\{w_{2}, h_{0}\right\}= & 2 k_{2}-\alpha \omega_{0} j\left[\sin \Omega t+\frac{1}{2} \sin (\Omega t+2 \phi)+\frac{1}{2} \sin (\Omega t-2 \phi)\right] \\
& +\frac{j^{3}}{\omega_{0}}\left(\frac{17}{32}+\frac{51}{16} \cos 2 \phi+\frac{3}{4} \cos 4 \phi-\frac{1}{8} \cos 6 \phi\right) .
\end{aligned}
$$

The analysis of the last four terms was done in the previous section. Since (4.3.4) is linear in $w_{2}$ and $k_{2}$, we can find $w_{2}$ and $k_{2}$ by analyzing the equation

$$
\frac{\partial w_{2}}{\partial t}+\left\{w_{2}, h_{0}\right\}=2 K_{2}-\alpha \omega_{0} j\left[\sin \Omega t+\frac{1}{2} \sin (\Omega t+2 \phi)+\frac{1}{2} \sin (\Omega t-2 \phi)\right] \text {. }
$$

Adding the results of this analysis to the $\mathrm{w}_{2}$ and $\mathrm{K}_{2}$ of the previous section will give the total $\mathrm{w}_{2}$ and $\mathrm{k}_{2}$.

In analyzing $(4.3 .5)$ we first set $k_{2}=0$ and integrate to find $w_{2}$.

This gives

$$
w_{2}=+\alpha \omega_{0} j\left(\frac{\cos \Omega t}{\Omega}+\frac{1}{2} \frac{\cos (\Omega t+2 \phi)}{\left(\Omega+2 \omega_{0}\right)}+\frac{1}{2} \frac{\cos (\Omega t-2 \phi)}{\left(\Omega-2 \omega_{0}\right)}\right) .
$$

We see that $\omega_{2}$ is very large if either $\Omega, \Omega+2 \omega_{0}$, or $\Omega-2 \omega_{0}$ is small.

This is the familiar problem of small denominators. For $\Omega>\omega_{0}$ we pick $w_{2}$ as in (4.3.6), but we must treat the other cases individually.

Here we treat the case where $\Omega \sim 2 \omega_{0}$. Then we choose $K_{2}$ to cancel the only term which we can not transform away. Thus we have 


$$
\begin{aligned}
& w_{2}=\alpha \omega_{0} j\left(\frac{\cos \Omega t}{\Omega}+\frac{1}{2} \frac{\cos (\Omega t+2 \phi)}{\Omega+2 \omega_{0}}\right) \\
& k_{2}=\frac{\alpha \omega_{0} j}{4} \sin (\Omega t-2 \phi) .
\end{aligned}
$$

Collecting all the terms in $\mathrm{K}$ including those parts of $\mathrm{K}_{2}$ calculated in the previous section we have

$$
K=\omega_{0} j+\frac{3}{2} \varepsilon j^{2}-\frac{17}{64} \varepsilon^{2} \frac{j^{3}}{\omega_{0}}+\frac{\alpha \omega_{0} j}{4} \sin (\Omega t-2 \phi) .
$$

In this case we have not been able to transform to a Hamiltonian independent of the angle, but we have simplified the analysis by eliminating two terms. In fact, this Hamiltonian can now be solved by transforming to the new variables

$$
\begin{aligned}
& I=j \\
& \theta=\frac{\Omega}{2} t+\phi .
\end{aligned}
$$

Using this transformation $K$ becomes a time independent one degree of freedom. Hamiltonian,

$$
K=\omega_{0} I+\frac{3}{2} \varepsilon I^{2}-\frac{17}{64} \varepsilon^{2} \frac{I^{3}}{\omega_{0}}+\frac{\alpha \omega_{0} I}{4} \sin 2 \theta,
$$

which can now be solved by quadrature. 


\subsection{Superconvergence; Kolmogorov's Technique}

In this section we discuss the phenomenon of superconvergence. This phenomenon is known to occur when one is doing Hamiltonian perturbation theory using the generating functions of mixed variables $F(\underset{\sim}{q}, \underset{\sim}{P}, t)$. Here we show how the same phenomenon occurs when doing Hamiltonian perturbation theory with Lie transforms.

In the Poincare-Von Zeipel technique $[4,5]$ a mixed variable generating function $F(q, P, t)$ is introduced and chosen, order by order, to cancel the phase dependent part (rapidly varying part) of the Hamiltonian. Kolmogorov [6] improved this scheme by introducing the method of successive transformations, here after known as Kolmogorov's technique. In this scheme one transfoms to successive systems. After each transformation the Hamiltonian becomes solvable to higher order. The improvement arising in this technique is that after doing the $\mathrm{n}^{\text {th }}$ transformation one has solved the problem to order $\varepsilon^{(2 n)}$. Another way of putting it is as follows. Let $\delta h_{n}$ be the rapidly varying part of the Hamiltonian after the $\mathrm{n}^{\text {th }}$ transformation. Then the terms of the sequence $\delta h_{1}, \delta h_{2}, \delta h_{3} \ldots$ are of orders $\varepsilon^{2}, \varepsilon^{4}, \varepsilon^{8}, \ldots$. This phenomenon is termed superconvergence. It does not by itself imply convergence.

In this paper we show how superconvergence arises in Lie transform theory. We show explicitly that the successive errors go like $\varepsilon^{2}, \varepsilon^{4}, \varepsilon^{8}, \ldots$ when combining Lie transform theory with Kolmogorov's technique. This has been shown previously in the case of time independent transformations [9]. Here we extend these results to the case of time dependent transformations. We also draw new conclusions concerning the use of Kolmogorov's technique in practical calculations. 
We begin by assuming we have an ordered Hamiltonian which is solvable In zeroth order.

$$
h(\underset{\sim}{z}, t)=\sum \varepsilon^{n_{h}} h_{n}(\underline{z}, t) .
$$

Transforming to appropriate coordinates, the zeroth order Hamiltonian is . written entirely in terms of the momenta.

$$
h_{0}(\underset{\sim}{z}, t)=h_{0}(p) \text {. }
$$

As usual, the vector $z$ represents all of the phase space variables.

We will introduce successive Lie transforms to successive new Hamiltonians. We distinguish the various transforms by a left subscript. In particular, we introduce the transform ${ }_{1} T$. According to the usual method, the variable mapping is given by

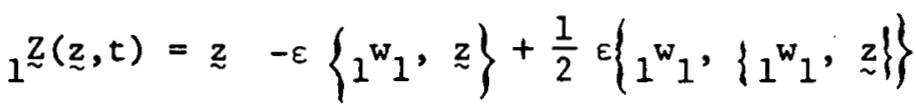

$$
\begin{aligned}
& -\frac{1}{2} \varepsilon\left\{w_{1}, z=+\ldots\right. \\
& =1^{\mathrm{T}} \underset{\sim}{z},
\end{aligned}
$$

where the ${ }_{1} \mathrm{w}_{i}$ 's are yet unchosen.

Following the general Lie transform scheme, we choose the generating function $1^{w_{i}}$ by examining the equations for the transformed Hamiltonian ${ }_{1} K=\sum_{n} \varepsilon^{n}{ }_{1} K_{n}(z, t)$. To second order these equations are

$$
\begin{aligned}
& { }_{1} \mathrm{~K}_{0}=h_{0} \\
& \frac{\partial{ }_{1} \mathrm{w}_{1}}{\partial t}+\left\{\mathrm{w}_{1}, \mathrm{~h}_{0}\right\}={ }_{1} \mathrm{~K}_{1}-\mathrm{h}_{1} \\
& \frac{\partial \mathrm{w}_{2}}{\partial t}+\left\{\mathrm{w}_{2}, \mathrm{~h}_{0}\right\}=2\left(\mathrm{~K}_{2}-\mathrm{h}_{2}\right)-\left\{\mathrm{w}_{1}, \mathrm{~h}_{1}+{ }_{1} \mathrm{~K}_{1}\right\}:
\end{aligned}
$$

In the ordinary Lie transform method, we choose the new Hamiltonian ${ }_{1} \mathrm{~K}$ order by order to make the right hand side of these equations have zero average along a particle orbit. Then the ${ }_{1}{ }^{w}{ }^{\prime} s$, which are found by integration of the right hand side along an orbit of $h_{0}$, will be nonsecular. 
In the successive transformation scheme, we choose the new Hamiltonian differently. The general rule is that we calculate the generating function only in those orders which can be done nonrecursively. In particular, in first order we can pick; ${ }_{1} K_{1}=\bar{h}_{1}$ (overbar denotes orbit average) and find $1_{1} w_{1}$ to calculate the Poisson bracket on the right hand" side of (4.4.6). Hence. we choose $1_{1} w_{r}=0$ for $n^{\prime}>1$, and we use $1^{w}$ and the transformation equations, e,g. $(4.4 .6)$, to find ${ }_{1} K_{n}$ for $n>1$.

The result is that we now have the hamiltonian ${ }_{1} \mathrm{~K}$ which is solvable to first order. ${ }_{1} K_{1}={ }_{1} K_{1}(p)$ since ${ }_{1} K_{1}$ was chosen to be the average of $h_{1}$. We want to apply a second transformation, but before doing so, we rearrange the terms in our Hamiltonian. We pick our "new-old" Hamiltonian. $1^{h}=\sum \varepsilon^{n}{ }_{1}{ }_{n}$ to be given by

$$
\begin{aligned}
& { }_{1} \mathrm{~h}_{0}={ }_{1} \mathrm{~K}_{0}+\varepsilon_{1} \mathrm{~K}_{1} \\
& { }_{1} \mathrm{~h}_{1}=0 \\
& { }_{1} \mathrm{~h}_{2}={ }_{1} \mathrm{~K}_{2} \\
& { }_{1} \mathrm{~h}_{3}={ }_{1} \mathrm{~K}_{3} .
\end{aligned}
$$

That is, we absorb all of the solvable parts in the unperturbed Hamiltonian.

Now we apply a second transformation $2^{T}$ to the above Hamiltonian. Since there is no first order perturbation to transform away, $2^{T}$ vanishes in first order. Using this fact, we get the following equations relating the new Hamiltonian to the generating function of $2^{T}$.

$$
\begin{aligned}
& \frac{\partial 2^{w_{2}}}{\partial t}+\left\{{ }_{2} w_{2},{ }_{1} h_{0}\right\}=2\left({ }_{2} K_{2}-{ }_{1} h_{2}\right) \\
& \frac{\partial{ } w_{3}}{\partial t}+\left\{{ }_{2} w_{3},{ }_{1} h_{0}\right\}=3\left({ }_{2} K_{3}-{ }_{1} h_{3}\right)
\end{aligned}
$$




$$
\frac{\partial 2^{w}}{\partial t}+\left\{{ }_{2} w_{4},{ }_{1} h_{0}\right\}=4\left({ }_{2} K_{4}-{ }_{1} h_{4}\right)-\left\{{ }_{2} w_{2},{ }_{1} h_{2}+{ }_{2} K_{2}\right\}
$$

Now we pick $2^{K}$ so that the right hand sides have zero orbit average along $1 \mathrm{~h}_{\mathrm{o}}$. We note that Eqs. (4.4.11) and (4.4.12) can be solved nonrecursively, but to solve $(4.4 .13)$, we must first know the results from $(4.4 .11)$. Hence we select $2_{2} \mathrm{w}_{2}$ and $2^{\mathrm{w}_{3}}$ by the usual method, we set $2^{\mathrm{w}} \mathrm{n}=0$ for $\mathrm{n}>3$, and we use the higher order transform equations to find $2_{n}$ for $n>3$.

We remark on the ordering system here. By regrouping terms in (4.4.7), we have messed up the ordering scheme. We have added an order $\varepsilon^{1}$ term to an order $\varepsilon^{0}$ term and cailed the result order $\varepsilon^{0}$. This does not invalidate our results. When we calculate $2^{\mathrm{w}_{2}}$ in (11) it is good to second order, but since ${ }_{1} h_{0}$ has a first order part, $2_{2} w_{2}$ has higher than $\varepsilon^{2}$ effects included in it.

Let's review what has been accomplished. First we introduced an order $\varepsilon$ transformation ${ }_{1} T$. Then we regrouped terms in the Hamiltonian and transformed away order $\varepsilon^{2}$ and order $\varepsilon^{3}$ oscillations using $2^{T}$. If we regroup terms again, we will have $2_{n} h_{n}=0$ for $n=1,2,3$. It follows that the perturbations in $2^{\text {h }}$ are of order $\varepsilon^{4}$.

To construct an induction proof we use the equation (4.1.14). We assume the unsolvable terms in the Hamiltonian $n-1^{\text {h }}$ are of orders higher than or equal to $\varepsilon^{\left(2^{n}\right)}$. By regrouping terms we get $n-1^{h}=0$ for $1 \leq m<2^{n}$. We apply the transformation $n^{T}$. We must have $n^{w_{1}}, \cdots, n^{w}{ }_{\left(2^{n}-1\right)}=0$; $n^{\mathrm{T}}, \ldots, \mathrm{n}^{\mathrm{T}}{\left(2^{\mathrm{n}}-1\right)}=0$; and $\mathrm{n}_{1}, \ldots, \mathrm{n}^{\mathrm{K}}{ }_{\left(2^{\mathrm{n}}-1\right)}=0$. Simply put, if there is no perturbation to $\left(2^{n}-1\right)^{\text {th }}$ order, then there is no new transformation nor new Hamiltonain to $\left(2^{n}-1\right)^{\text {th }}$ order. From (4.1.14), this 1mplies

$$
\left.\frac{\partial_{n m} w_{m}}{\partial t}+\left\{w_{m}, h_{0}\right\}=m \dot{x}_{n} K_{m}-{ }_{n-1} h_{m}\right)
$$


for $m<2^{n+1}-1$, meaning that we can choose our transformation nonrecursively to order $\varepsilon^{\left(2^{n+1}\right)}$ or higher. This completes the induction proof.

Kolmogorov's technique is very appealing since the errors in the perturbation theory are of orders $\varepsilon, \varepsilon^{2}, \varepsilon^{4}, \varepsilon^{8}, \ldots$ after each step rather than the usual $\varepsilon, \varepsilon^{2}, \varepsilon^{3}, \varepsilon^{4}, \ldots$. One should be closer to the right answer after $n$ steps of Kolmogorov's technique than after $n$ steps of the usual method. To theoretical physicists Kolmogorov's technique is appealing for another reason. In calculating the orbit integrals Kolmogorov's technique uses an improved unperturbed Hamiltonian. The inclusion of higher order terms in the unperturbed Hamiltonian is called renomalization, and it is believed to be important in a number of fields such as quantum field theory, statistical mechanics, and plasma physics.

A point to be noted about Kolmogorov's technique is that terms of order $\varepsilon^{3}, \varepsilon^{5}, \varepsilon^{6}, \ldots$ do not vanish. Instead they are being absorbed in lower order terms. For example, the term of order three is included in the generating function $2^{\mathrm{w}}=2_{2} \mathrm{w}_{2}+\varepsilon_{2} \mathrm{w}_{3}$. Although Kolmogorov's technique has fewer terms than the standard method, each term is much more complicated.

There are reasons for believing that Kolmogorov's technique is not the most practical method of attacking problems. To see one reason, compare Kolmogorov's technique to the standard Deprit method in fourth order theory. Kolmogorov's technique requires knowing $2^{\mathrm{h}}\left({ }_{2} \mathrm{~h}\right.$ o plus perturbations) at this point, and $2^{h}$ must be found by multiplying three infinite series: $2^{\mathrm{h}}=2^{\mathrm{T}^{-1}} \cdot 1^{\mathrm{T}^{-1} \cdot \mathrm{h}}$. In the standard Deprit method, only two infinite series are ever multiplied, c.f. (4.1.14).

Another criticism of Kolmogorov's technique is that it can introduce spurious small denominators. An example of this is the one degree of freedom Hamiltonian $h=\frac{1}{2} p^{2}+\frac{1}{2} \omega_{0}^{2} q^{2}+\Delta U(\varepsilon, q)$ for which the transformed Hamiltonian 
is exponential in the true action $\mathrm{J}$ :

$$
\mathrm{K}(\mathrm{J})=\omega_{0}\left(1-\mathrm{e}^{-\varepsilon J}\right) / \varepsilon=\omega_{0} J-\frac{\varepsilon}{2} \omega_{0} J^{2}+\ldots .
$$

If we use perturbation theory to find $K$ from $h$ (as in section 4.2), we would have $\mathrm{K}$ to first order in $\varepsilon$ upon doing the first order transformation. Using the standard ...method we have a fixed ordering, and so we just generate the power serles in $(4.4 .15)$ term by term. In Kolmogorov's technique we change the ordering by absorbing the solvable terms in the unperturbed Hamiltonian. After the first transformation we would get

$$
{ }_{1} h_{0}(j)=\omega_{o} j-\frac{\varepsilon}{2} \omega_{o} j^{2} \text {. }
$$

Recalling section 4.2 , we note that each time we integrated along a particle orbit we had a $1 / \omega_{0}$ in our expressions for the $w_{n}$ 's. If we instead use the unperturbed Hamiltonain of $(4.4 .16)$, we end up with a factor of $1 /{ }_{1} \omega_{0}=$ $1 / \omega_{0}=1 /\left(\omega_{0}-\varepsilon \omega_{0} j\right)$. We see that Kolmogorov's technique fails for $j=1 / \varepsilon$ and perhaps beyond, because it gives a small denominator.

The reason Komogorov's technique is worse than the standard method in this case is not hard to see. The improved frequency ${ }_{1} \omega_{0}$ is closer than $\omega_{0}$ to the final result $\omega$ in the range $0<J \leqslant 2 / \varepsilon$, but the improved frequency is much farther than $\omega_{0}$ from the final result for $2 / \varepsilon \leq J<\infty$. Furthermore the improved frequency is qualitatively different: it has a root for finite J. Neither $\omega_{0}$ nor $\omega$ are ever zero in the range $0 \leq J<\infty$.

We conclude that Kolmogorov's technique is not necessarily the most practical way of obtaining answers. This does not contradict the fact that Kolmogorov [6], Arnold [20], and Moser [21] all used this technique to show convergence of certain invariant tori. In their proof the concern was about getting very good expression in small regions of phase space. In the interval $0 \leq \mathrm{J} \leq 1$, the improved Hamiltonian ${ }_{1} \mathrm{~h}$ o does give a much better value for the frequency than does the unperturbed Hamiltonian $h_{0}=\omega_{0} j$. 


\section{Discussion of the Choice of Conventions}

The primary purpose of this paper has been to give an expository account of Hamiltonian perturbation theory using the Lie transformation theory of Dewar, and from it to derive Deprit's perturbation theory. Furthermore, we have illustrated the theories with examples. However, a secondary purpose of this paper has been to introduce conventions which are convenient in practical applications. In this section we outline how and why the conventions presented here differ from previous ones.

We first mention that there are two minus signs in the definition of the operator $\mathrm{L}_{\mathrm{f}}$. The more obvious one is that (2.1.8) differs from the corresponding definttions in for example references [8] and [10]: We chose the definition (2.1.8) to rid Jacobi's identity of a minus sign. In the case where $L_{f}$ is defined by

$$
L_{f} g=\{g, f\},
$$

Jacobi's identity is

$$
\left[\mathrm{L}_{\mathrm{f}}, \mathrm{L}_{\mathrm{g}}\right]=-\mathrm{L}_{\{\mathrm{f}, \mathrm{g}\}}
$$

A second minus sign occurs because of a difference in the meanings of coordinates and momenta in the different literatures. In the celestial mechanics 11terature [7-9] the unperturbed Hamiltonian depends only on the coordinates. Here and elsewhere $[1,16,18,19]$ the unperturbed Hamiltonian is chosen to depend only on the momenta. This is the natural choice when dealing with nearly free particle motion where momentum is conserved in the unperturbed system. Of course, when momenta and coordinates are interchanged, the sign of the Poisson bracket changes. 
In choosing the transformation $T$ in terms of $L_{W}$, there are a number of possibilities. Hori [7] defines $T$ by simple exponentiation: $T \equiv \exp \left(L_{w}\right)$. Deprit's choice [8], that $\mathrm{T}$ satisfies a differential equation, proves more useful in two respects. One is able to derive recursion relations for the successive terms, and one is able to do time dependent transformations in "a straightforward manner. With Hori's choice one does time dependent theory by first enlarging the phase space with time as a new coordinate. Dewar [10] improved Deprit's work by not requiring $T$ to be a power series. Basically, we have followed Dewar's formalism, but we differ in minor ways on the form of the differential equation. Our choice is to make the evolution of $T$ in $\theta$ by $\mathrm{L}_{w}$ exactly analogous to the evolution of the time development operator $M$ in $t$ by $L_{h}$.

Another convention we must choose is whether $T$ should transform from old to new coordinates as done here, or from new to old as in [10]. The choice made here simplifies the perturbation equations, in particular Eq. (4.1.14). In perturbation theory we average (4.1.14) along a particle orbit to find $K_{n}$. Since the $K_{n}$ 's are averages and the $w_{n}$ 's have no averages, this gives

$$
\mathrm{K}_{\mathrm{n}}=\overline{\mathrm{h}}_{\mathrm{n}}+\sum_{\mathrm{m}=1}^{\mathrm{n}-1} \frac{\mathrm{m}}{\mathrm{n}} \overline{\mathrm{T}_{\mathrm{n}-\mathrm{m}}^{-1} \mathrm{~h}_{\mathrm{m}}} \text {. }
$$

Had we made the opposite choice, we would have effectively interchanged $h$ and $k$, and the result for $k_{n}$ would be

$$
K_{n}=\bar{h}_{n}-\frac{1}{n} \sum_{m=1}^{n-1}\left(\overline{L_{n-m}} h_{m}+m \overline{T_{n-m}^{-1} \cdot K_{m}}\right) \text {. }
$$

By our choice, we avoid the extra set of terms in (5.1').

We also write our expansions differently. In previous works, the expansion $(4.1 .4)$ is of the form 


$$
w=\sum_{n=0} \frac{1}{n !} \theta^{n}{ }_{n+1}
$$

That cholce does not allow Eq. (4.1.14) to have such a simple appearance. The effect of that choice is to insert $n$ !'s in all the equations. The result is that Deprit's equations (e.g. ref. [8] eq. (27)) have binomial coefficients whereas ours have simple fractions.

In summary, we have changed the conventions in minor ways. We have presented the conventions we have found to be most useful in practical calculations.

\section{ACKNOWLEDGEMENTS}

The author thanks A. N. Kaufman and R. G. Littlejohn for many comments on the first draft of this work. These comments did much to shape the present version. 


\section{APPENDIX A}

\section{Notations for Derivatives}

The derivative notation used in this paper has been chosen to be very explictt, and as a result it is detailed and cumbersome. In the usual physics use of derivatives, such a detalled notation is not needed, and one may wonder if such a cumbersome notation is needed. In this appendix we show how confusion can arise if one uses a less explicit notation.

We first consider two functions, $f$ and $F$, which obey the following relation,

$$
f(\underset{\sim}{z}, t, \theta)=F(\underset{\sim}{z}(z, t, \theta), t) .
$$

In our discussion of Lie transforms, we need to differentiate equations such as (Al) with respect to $z_{\mathrm{m}}$. In the usual physics notation this would be written

$$
\frac{\partial f}{\partial z_{m}}=\sum_{n} \frac{\partial F}{\partial Z_{n}} \frac{\partial z_{n}}{\partial z_{m}} .
$$

We know what $\frac{\partial F}{\partial Z}$ means in this case, but we need an interpretation of this 8ymbol for other cases.

The two most common interpretations of the symbol $\frac{\partial F}{\partial Z}$ are:

A. F is always a function of $\underset{\sim}{z}$, so $\frac{\partial F}{\partial z}$ is simply the derivative of $F$ with respect to its argument.

B. Given a symbol such as $\frac{\partial F}{\partial Z}$, the object below the line (in this case $Z$ ) denotes both the argument of the function and the variable with respect to which the derivative is taken.

Let us first consider A. This interpretation cannot be used in Lie transform discussions, since in these discussions we approximate (A1) by 


$$
\begin{aligned}
& \mathbf{f}(\underset{\sim}{z}, \tau, \theta) \doteq \bar{F}(\underset{\sim}{z} ; t)+\dot{\theta}\left\{w_{1}, F^{\prime}\right\} \\
& \therefore \quad F(\underset{\sim}{z}, t)+\theta\left[\frac{\partial w_{1}}{\partial q} \cdot \frac{\partial F}{\partial p} \cdot \frac{\partial w_{1}}{\partial \underset{\sim}{p}} \cdot \frac{\partial F}{\partial q}\right] .
\end{aligned}
$$

The derivatives which appear in (A3) mean

$$
\frac{\partial F}{\partial Z,}=\frac{\partial}{\partial z}[F(\underset{z}{z}, t)] \text {, }
$$

whereas, using interpretation $A$, we would assign them the meaning

$$
\frac{\partial \ddot{F}}{\partial z} \stackrel{?}{=} \frac{\partial}{\partial z}[F(z(z, t, \theta), t)] \text {. }
$$

Thus we cannot use interpretation $A$ in Lie transform discussions.

Now we consider interpretation B. This interpretation is ambiguous when we consider derivatives of the form

$$
\frac{\partial F}{\partial t}
$$

Sirice $t$ (the object below the line) doesn't indicate $\underset{\sim}{z}$ or $z$, we don't know whether (A7) means

or

$$
\frac{\partial}{\partial \dot{t}}(F(\underset{\sim}{z}, t))
$$

$$
\frac{\partial}{\partial t}(F(\underset{\sim}{z}(\underset{\sim}{z}, t ; \theta), i t))
$$

or even.

$$
\begin{aligned}
& \frac{\partial}{\partial t}(F(\underset{\sim}{z}, t)) \text { evaluated at } \\
&\underset{\sim}{z}=\underset{\sim}{\underset{\sim}{z}, t}, \theta)
\end{aligned}
$$

It is important to distinguish between these three objects in the expansion of (A1) using the chain rule, which might be written in the following way:

$$
\begin{aligned}
\frac{\partial}{\partial t} \cdot(F(\underset{\sim}{z}(\underset{\sim}{z}, t, \theta), t)) & =\sum_{k} \frac{\partial F}{\partial z_{k}} \cdot \frac{\partial z_{k}}{\partial t} \\
& +\frac{\partial}{\partial t}(F(\underset{z}{z}, t)) \begin{array}{l}
\text { evaluated } a t \\
\underset{\sim}{z}=\underset{\sim}{2} \underset{\sim}{z}, t, \theta) .
\end{array}
\end{aligned}
$$


Though the notation used in (A10) is clear in this instance, it is cumbersome.

So we see that the standard notation is ambiguous when using lie

transforms, and we do not use it. Instead we use the notation introduced

In Chapter 2 which is unambiguous though cumbersome. 


\section{References}

II] E. J. Saletan and A. H. Cromer, Theoretical Mechanics, (John Wiley and Sons, Inc., New York, 1971).

[2] E. T. Whittaker, A Treatise on the Analytical Dynamics of Particles and Rigid Bodies, (Dover Publications, New York, 1944).

[3] H. Goldstein, Classical Mechanics, (Addison-Wesley Publish1ng Co., Reading, Mass., 1950).

[4] H. Poincare, Les Méthodes Nouvelles de la Mécanique Celeste, Vols. 1-3, 1892, 1893, 1899 (English Translation, NASA, Washington, 1967).

[5] A. Nayfeh, Perturbation Methods, (John Wiley and Sons, New York, 1973).

[6] A. N. Kolmogorov, Dok1. Akad. Nauk. SSSR 98, 527 (1954).

[7] G. Hori, Pub. Astron. Soc. Jap. 18, 287 (1966).

[8] A. Deprit, Cel. Mech. 1, 12 (1969).

[9] R. A. Howland, Cel. Mech. 15, 327 (1977).

[10] R. L. Dewar, J. Phys. A 9, 2043 (1976).

[11] R. L. Dewar; J. Phys. A 11, 9 (1978).

[12] J. J. Sakurai, Advanced Quantum Mechanics, (Addison-Wesley Publishing Co., Reading, Mass., 1967).

[13] E. G. C. Sudarshan and N. Mukunda, Classical Dynamics: A Modern Perspective, (John Wiley and Sons, New York, 1974).

[14] R. Abraham, Foundations of Mechanics, (W. A. Benjamin, Inc., New York, 1967).

[15] J. L. Anderson, Am. J. Phys. 40, 541 (1972).

[16] J. R. Cary, J. Math Phys. 18, 2432 (1977).

[17] R. G. Littlejohn, private communication. 
[18] G. R. Smith, Ph.D. thesis, University of California, Berkeley (1977).

[19] B. V. Chtrikov, "A Universal Instability of Many-Dimensional Oscillator Systems", Physics Reports, to be published.

[20] V. I. Arnold, Usp. Mat. Nauk. 18, 91 (1963).

[21] J. Moser, Nachr. Akad. Wiss., Gottingen, Math. Phys. K1., No.1, I (1962). 